\title{
Numerical Analysis of a High-Order Scheme for Nonlinear Fractional Differential Equations with Uniform Accuracy
}

\author{
Junying $\mathrm{CaO}^{1}$ and Zhenning $\mathrm{Cai}^{2, *}$ \\ ${ }^{1}$ School of Data Science and Information Engineering, Guizhou Minzu \\ University, 550025 Guiyang, China \\ ${ }^{2}$ Department of Mathematics, National University of Singapore, Singapore \\ 119076, Singapore
}

Received 7 March 2020; Accepted (in revised version) 28 May 2020

\begin{abstract}
We introduce a high-order numerical scheme for fractional ordinary differential equations with the Caputo derivative. The method is developed by dividing the domain into a number of subintervals, and applying the quadratic interpolation on each subinterval. The method is shown to be unconditionally stable, and for general nonlinear equations, the uniform sharp numerical order $3-\nu$ can be rigorously proven for sufficiently smooth solutions at all time steps. The proof provides a general guide for proving the sharp order for higher-order schemes in the nonlinear case. Some numerical examples are given to validate our theoretical results.
\end{abstract}

AMS subject classifications: 65M06

Key words: Caputo derivative, fractional ordinary differential equations, high-order numerical scheme, stability and convergence analysis.

\section{Introduction}

In the past decades, fractional differential equations have been studied extensively by many researchers, due to its success in describing some physical phenomena and chemical processes more accurately than integer order differential equations $[18,30$, $33,34]$. Like most classical differential equations, the exact solutions of fractional order differential equations are usually not available to us. Even if analytical solutions can be found, they usually appear in the form of series and are difficult to evaluate. Therefore, the numerical study of fractional differential equations has also inspired a number of excellent research works such as $[6,7,12,13,15,21,29,41]$.

\footnotetext{
${ }^{*}$ Corresponding author. Email addresses: caojunying@gzmu.edu.cn (J. Cao), matcz@nus.edu.sg (Z. Cai) 
In this work, we are interested in the following initial value problem: For some $\nu \in(0,1)$, we would like to find $y(x)$ such that

$$
{ }_{0} D_{x}^{\nu} y(x)=f(x, y(x)), \quad 0<x \leq T
$$

subject to the initial condition $y(0)=y_{0}$. In (1.1), the operator ${ }_{0} D_{x}^{\nu}$ is the Caputo derivative, defined by

$$
{ }_{0} D_{x}^{\nu} y(x)=\int_{0}^{x} \omega_{1-\nu}(x-s) y^{\prime}(s) d s
$$

where $\omega_{1-\nu}$ is defined by

$$
\omega_{1-\nu}(x)=\frac{x^{-\nu}}{\Gamma(1-\nu)}
$$

with $\Gamma(\cdot)$ being Euler's gamma function. The function $\omega_{1-\nu}(x)$ acts as the convolutional kernel, which satisfies

$$
\int_{s}^{t} \omega_{\nu}(t-\mu) \omega_{1-\nu}(\mu-s) d \mu=\omega_{1}(t-s)=1, \quad \forall 0<s<t<+\infty .
$$

The numerical method for this equation has been extensively studied in the context of linear partial differential equations. For example, the L1-type schemes based on piecewise linear interpolation have been studied in $[5,10,27]$, where the numerical order is $2-\nu$. In [1], the second-order L2-1 $\sigma$ method is proposed by quadratic interpolation. To achieve the sharp order $3-\nu$ for smooth solutions, one can use the L1-2 method proposed in [11], which is also based on the quadratic interpolation, or the method based on Taylor expansion as introduced in [20]. For this numerical order, fast numerical schemes to discretize the Caputo derivative is proposed in [39]. Generalization to $(r+1-\nu)$-th order schemes have been studied in $[3,23]$ by Lagrange interpolation. A common problem in these methods is that the theoretical order of the solution at the first time step can only achieve $2-\nu$, as is shown in the numerical analysis in [20]. Such a problem is also mentioned in [24], where the author uses a finer grid near the initial value to maintain the numerical accuracy. In [32], it is found that the size of the finer grid should be proportional to $\Delta t^{2-\nu}$, which may cause significant additional computational cost especially when $\nu$ is small. Moreover, it is pointed out in [16] that the realistic solution $u$ is usually nonsmooth at $t=0$, and the initial layer can also cause the reduction of numerical order. To solve this issue, graded meshes have been introduced to restore the numerical order $[5,36]$. In this case, the size of the finer grid could be even smaller if a high-order scheme is needed. Therefore, we are motivated to find a scheme that does not require a finer mesh for the first time step. For simplicity, in this paper we restrict ourselves to the case of a uniform mesh, and assume the smoothness of the solution. Other related works include, but not limited to $[2,8,38]$.

In principle, these methods can be directly generalized to nonlinear problems. However, the analysis of convergence order on such methods for nonlinear problems is less seen in the literature. In [4], the authors converted the Caputo fractional derivative 
to the Volterra integral and proved the order of accuracy $3+\nu$ for $0<\nu<1$ and 4 for $\nu \geq 1$. A similar technique is applied in [35]. In [17], the authors applied the L1 formula to the subdiffusion equation, and obtained the numerical order $\nu$ due to the insufficient smoothness of the solution. The numerical order $2-\nu$ is proven in $[22,26]$. A special cubic nonlinear term is studied in [37] to obtain a second-order numerical method. However, theoretical proofs of numerical schemes with order $3-\nu$ for general nonlinear problems are rarely seen in the literature. In [31], it is demonstrated that the generalization of schemes with order $3-\nu$ for linear problems also works for nonlinear problems, but the proof for nonlinear problems is given only for the truncation error. Clearly, nonlinearity has caused significantly difficulty in the numerical analysis, especially on the transition from the estimation of the truncation error to the error of the solution.

The aim of this work is to introduce a new $(3-\nu)$-th order scheme for the fractional differential equation (1.1). Our main contributions include:

- A new finite-difference approximation of the Caputo derivative is developed, which leads to a high-order numerical method for (1.1) with uniform accuracy at all time steps.

- The unconditional stability for the linear problem is proven rigorously.

- A novel proof for the convergence order is proposed for the general nonlinear right-hand sides.

Our method is based on the block-by-block approach [14,19] commonly used for integral equations $[28,40]$. To avoid degeneracy (order reduction) at the first time step, the proposed scheme couples the solutions at first two time steps, so that no smaller time steps are needed to achieve the sharp numerical order. Such coupling is not required in the later steps. The analysis of stability is complicated by these initial steps, which requires close look at the structure of the solutions. The convergence analysis is based on a novel technology that couples the idea of a recent work [25] and the strategy we used in the proof of stability, so that the order $3-\nu$ can be achieved for sufficiently smooth solutions and general nonlinear right-hand sides. The reference [25] provides a general framework which may be helpful to prove the numerical order for a number of high-order schemes such as $[1,11]$. In this paper, we follow the general steps therein with some alteration to the initial steps.

The rest of this paper is organized as follows. Our numerical scheme is introduced in Section 2. In Section 3, we prove the unconditional stability of our method. Section 4 is devoted to the proof of the convergence order, as is verified by our numerical examples in Section 5. Finally, some concluding remarks are given in Section 6.

\section{A finite difference approximation to the Caputo derivative}

In this section, we will construct an efficient numerical scheme for the problem (1.1). For simplicity, we consider a uniform grid on $[0, T]$ defined by the grid points 
$x_{j}=j \Delta x, j=0, \ldots, 2 N$, where $N$ is a positive integer, and $\Delta x=\frac{T}{2 N}$ is the grid size. Below we are going to use the short hand $y_{j}=y\left(x_{j}\right)$ and $f_{j}=f\left(x_{j}, y_{j}\right)$ for all $j=0, \ldots, 2 N$.

First, we propose a high-order approximation to the Caputo derivative ${ }_{0} D_{x}^{\nu} y(x)$ on grid points $x_{i}$ based on piecewise quadratic interpolation. To present the quadratic interpolation, we introduce the following notation:

$$
I_{\left[x_{j}, x_{j+2}\right]} y(x)=\varphi_{0, j}(x) y_{j}+\varphi_{1, j}(x) y_{j+1}+\varphi_{2, j}(x) y_{j+2}, \quad j \in \mathbb{N},
$$

where $\varphi_{i, j}(x), i=0,1,2$, are Lagrange interpolating polynomials defined as

$$
\begin{aligned}
& \varphi_{0, j}(x)=\frac{1}{2 \Delta x^{2}}\left(x-x_{j+1}\right)\left(x-x_{j+2}\right), \\
& \varphi_{1, j}(x)=-\frac{1}{\Delta x^{2}}\left(x-x_{j}\right)\left(x-x_{j+2}\right), \\
& \varphi_{2, j}(x)=\frac{1}{2 \Delta x^{2}}\left(x-x_{j}\right)\left(x-x_{j+1}\right) .
\end{aligned}
$$

When $j=1,2$, we approximate ${ }_{0} D_{x}^{\nu} y\left(x_{j}\right)$ by ${ }_{0} D_{x}^{\nu}\left(I_{\left[x_{0}, x_{2}\right]} y\right)\left(x_{j}\right)$ :

$$
\begin{aligned}
{ }_{0} D_{x}^{\nu} y\left(x_{1}\right) & =\int_{0}^{x_{1}} y^{\prime}(s) \omega_{1-\nu}\left(x_{1}-s\right) d s \approx \int_{0}^{x_{1}}\left[I_{\left[x_{0}, x_{2}\right]} y(s)\right]^{\prime} \omega_{1-\nu}\left(x_{1}-s\right) d s \\
& =A_{1}^{0,0} y_{0}+A_{1}^{1,0} y_{1}+A_{1}^{2,0} y_{2}, \\
{ }_{0} D_{x}^{\nu} y\left(x_{2}\right) & =\int_{0}^{x_{2}} y^{\prime}(s) \omega_{1-\nu}\left(x_{2}-s\right) d s \approx \int_{0}^{x_{2}}\left[I_{\left[x_{0}, x_{2}\right]} y(s)\right]^{\prime} \omega_{1-\nu}\left(x_{2}-s\right) d s \\
& =A_{2}^{0,0} y_{0}+A_{2}^{1,0} y_{1}+A_{2}^{2,0} y_{2},
\end{aligned}
$$

where

$$
A_{j}^{i, 0}=\int_{0}^{x_{j}} \varphi_{i, 0}^{\prime}(s) \omega_{1-\nu}\left(x_{j}-s\right) d s, \quad i=0,1,2, \quad j=1,2 .
$$

To approximate ${ }_{0} D_{x}^{\nu} y\left(x_{j}\right)$ for $j>2$, we assume that the values of $y_{0}, y_{1}, \ldots, y_{j}$ are all given. Different approximations will be used for odd and even $j$. When $j=2 m+1$, we approximate $y(x), x \in\left[0, x_{2 m+1}\right]$ by

$$
y(x) \approx \begin{cases}I_{\left[x_{0}, x_{2}\right]} y(x), & \text { if } \quad x \in\left[x_{0}, x_{1}\right], \\ I_{\left[x_{2 k-1}, x_{2 k+1}\right]} y(x), & \text { if } \quad x \in\left[x_{2 k-1}, x_{2 k+1}\right], \quad k=1, \ldots, m .\end{cases}
$$

This suggests the following approach

$$
\begin{aligned}
{ }_{0} D_{x}^{\nu} y\left(x_{2 m+1}\right)= & \int_{0}^{x_{1}} y^{\prime}(s) \omega_{1-\nu}\left(x_{2 m+1}-s\right) d s \\
& +\sum_{k=1}^{m} \int_{x_{2 k-1}}^{x_{2 k+1}} y^{\prime}(s) \omega_{1-\nu}\left(x_{2 m+1}-s\right) d s \\
\approx & \int_{0}^{x_{1}}\left[I_{\left[x_{0}, x_{2}\right]} y(s)\right]^{\prime} \omega_{1-\nu}\left(x_{2 m+1}-s\right) d s
\end{aligned}
$$




$$
\begin{aligned}
& +\sum_{k=1}^{m} \int_{x_{2 k-1}}^{x_{2 k+1}}\left[I_{\left[x_{2 k-1}, x_{2 k+1}\right]} y(s)\right]^{\prime} \omega_{1-\nu}\left(x_{2 m+1}-s\right) d s \\
= & A_{2 m+1}^{0,0} y_{0}+A_{2 m+1}^{1,0} y_{1}+A_{2 m+1}^{2,0} y_{2} \\
& +\sum_{k=1}^{m}\left(A_{2 m+1}^{0, k} y_{2 k-1}+A_{2 m+1}^{1, k} y_{2 k}+A_{2 m+1}^{2, k} y_{2 k+1}\right),
\end{aligned}
$$

where

$$
\begin{array}{ll}
A_{2 m+1}^{i, 0}=\int_{0}^{x_{1}} \varphi_{i, 0}^{\prime}(s) \omega_{1-\nu}\left(x_{2 m+1}-s\right) d s, & i=0,1,2, \\
A_{2 m+1}^{i, k}=\int_{x_{2 k-1}}^{x_{2 k+1}} \varphi_{i, 2 k-1}^{\prime}(s) \omega_{1-\nu}\left(x_{2 m+1}-s\right) d s, & i=0,1,2, \quad k=1, \ldots, m .
\end{array}
$$

Similarly, when $j=2 m+2$, we approximate the Caputo derivative on $x_{j}$ based on the following piecewise quadratic interpolation of $y(x)$ :

$$
y(x) \approx I_{\left[x_{2 k}, x_{2 k+2}\right]} y(x), \quad \forall x \in\left[x_{2 k}, x_{2 k+2}\right], \quad k=0, \ldots, m .
$$

As a consequence, ${ }_{0} D_{x}^{\nu} y\left(x_{2 m+2}\right)$ can be approximated in the same way as (2.4), and the result is

$$
{ }_{0} D_{x}^{\nu} y\left(x_{2 m+2}\right) \approx \sum_{k=0}^{m}\left(A_{2 m+2}^{0, k} y_{2 k}+A_{2 m+2}^{1, k} y_{2 k+1}+A_{2 m+2}^{2, k} y_{2 k+2}\right),
$$

where

$$
A_{2 m+2}^{i, k}=\int_{x_{2 k}}^{x_{2 k+2}} \varphi_{i, 2 k}^{\prime}(s) \omega_{1-\nu}\left(x_{2 m+2}-s\right) d s, \quad i=0,1,2, \quad k=0, \ldots, m .
$$

In all cases, the Caputo derivative ${ }_{0} D_{x}^{\nu} y\left(x_{j}\right)$ is approximated by a linear combination of $y_{k}$. Furthermore, by straightforward calculation, it can be found that for any fixed $i, j$ and $k$, the coefficient $A_{j}^{i, k}$ is proportional to $\Delta x^{-\nu}$. Therefore we summarize (2.2)-(2.4) and (2.8) to write down them uniformly as

$$
{ }_{0} D_{x}^{\nu} y\left(x_{j}\right) \approx{ }_{0} D_{\Delta x}^{\nu} y_{j}
$$

where the newly introduced operator ${ }_{0} D_{\Delta x}^{\nu}$ is the discrete Caputo derivative defined by

$$
{ }_{0} D_{\Delta x}^{\nu} y_{j}=\left\{\begin{array}{lll}
\Delta x^{-\nu}\left(\widehat{D}_{0} y_{0}+\widehat{D}_{1} y_{1}+\widehat{D}_{2} y_{2}\right), & \text { if } j=1, \\
\Delta x^{-\nu}\left(\widetilde{D}_{0} y_{0}+\widetilde{D}_{1} y_{1}+\widetilde{D}_{2} y_{2}\right), & \text { if } j=2, \\
\Delta x^{-\nu} \sum_{k=0}^{2 m+1} D_{k}^{(m)} y_{k}, & \text { if } j=2 m+1, \quad m=1, \ldots, N-1, \\
\Delta x^{-\nu} \sum_{k=0}^{2 m+2} \bar{D}_{k}^{(m)} y_{k}, & \text { if } j=2 m+2, \quad m=1, \ldots, N-1 .
\end{array}\right.
$$


Here all the coefficients " $D$ "s are constants depending only on $\nu$, and their values can be computed analytically:

$$
\begin{aligned}
& \widehat{D}_{0}=\frac{3 \nu-4}{2 \Gamma(3-\nu)}, \quad \widehat{D}_{1}=\frac{2(1-\nu)}{\Gamma(3-\nu)}, \quad \widehat{D}_{2}=\frac{\nu}{2 \Gamma(3-\nu)}, \\
& \widetilde{D}_{0}=\frac{3 \nu-2}{2^{\nu} \Gamma(3-\nu)}, \quad \widetilde{D}_{1}=-\frac{4 \nu}{2^{\nu} \Gamma(3-\nu)}, \quad \widetilde{D}_{2}=\frac{\nu+2}{2^{\nu} \Gamma(3-\nu)}, \\
& \bar{D}_{0}^{(m)}=\frac{1}{\Gamma(3-\nu)}\left(-\frac{2-\nu}{2}\left[(2 m)^{1-\nu}+3(2 m+2)^{1-\nu}\right]-\left[(2 m)^{2-\nu}-(2 m+2)^{2-\nu}\right]\right), \\
& \bar{D}_{2 k}^{(m)}=\frac{1}{\Gamma(3-\nu)}\left(-\frac{2-\nu}{2}\left[(2 m-2 k)^{1-\nu}+6(2 m-2 k+2)^{1-\nu}+(2 m-2 k+4)^{1-\nu}\right]\right. \\
& \left.-\left[(2 m-2 k)^{2-\nu}-(2 m-2 k+4)^{2-\nu}\right]\right), \quad k=1, \ldots, m, \\
& \bar{D}_{2 k+1}^{(m)}=\frac{2}{\Gamma(3-\nu)}\left((2-\nu)\left[(2 m-2 k)^{1-\nu}+(2 m-2 k+2)^{1-\nu}\right]\right. \\
& \left.+\left[(2 m-2 k)^{2-\nu}-(2 m-2 k+2)^{2-\nu}\right]\right), \quad k=0, \ldots, m, \\
& D_{0}^{(m)}=\frac{1}{\Gamma(3-\nu)}\left(\frac{2-\nu}{2}\left[(2 m)^{1-\nu}-3(2 m+1)^{1-\nu}\right]-(2 m)^{2-\nu}+(2 m+1)^{2-\nu}\right), \\
& D_{1}^{(m)}=\frac{1}{\Gamma(3-\nu)}\left(-\frac{2-\nu}{2}\left[(2 m-2)^{1-\nu}+3(2 m)^{1-\nu}-4(2 m+1)^{1-\nu}\right]\right. \\
& \left.-(2 m-2)^{2-\nu}+3(2 m)^{2-\nu}-2(2 m+1)^{2-\nu}\right) \\
& D_{2}^{(m)}=\frac{1}{\Gamma(3-\nu)}\left(\frac{2-\nu}{2}\left[4(2 m-2)^{1-\nu}+3(2 m)^{1-\nu}-(2 m+1)^{1-\nu}\right]\right. \\
& \left.+2(2 m-2)^{2-\nu}-3(2 m)^{2-\nu}+(2 m+1)^{2-\nu}\right) \\
& D_{2 k}^{(m)}=\bar{D}_{2 k+1}^{(m)}, \quad D_{2 k-1}^{(m)}=\bar{D}_{2 k}^{(m)}, \quad k=2, \ldots, m, \quad D_{2 m+1}^{(m)}=\bar{D}_{2 m+2}^{(m)}=\frac{\nu+2}{\Gamma(3-\nu) 2^{\nu}} .
\end{aligned}
$$

Based on the approximation (2.10), the numerical scheme for (1.1) with initial condition $y(0)=y_{0}$ can be written as

$$
{ }_{0} D_{\Delta x}^{\nu} y_{j}=f\left(x_{j}, y_{j}\right), \quad j=1, \ldots, 2 N .
$$

The above scheme is implicit. Since ${ }_{0} D_{\Delta x}^{\nu} y_{1}$ depends on $y_{2}$, the values of $y_{1}$ and $y_{2}$ have to be solved simultaneously, which is the key to getting uniform accuracy without loss of precision at the first time step. For $j>2$, solving $y_{j}$ needs only to solve a single equation.

Remark 2.1. Our numerical scheme includes more coefficients than some other highorder schemes such as the L1-2 method [11] due to different discretizations on odd 
and even time steps. In fact, we can find from (2.11) that the difference between odd and even time steps exists only in the first few coefficients with subscripts $0,1,2$. In particular, the coefficients $D_{2 m+1}^{(m)}$ and $\bar{D}_{2 m+2}^{(m)}$ are the same for all $m$, indicating that the numerical scheme for the nonlinear solver stays the same throughout the evolution of the solution except for the first two time steps.

\section{Stability analysis}

This section is devoted to the stability analysis of our numerical scheme. Consider the fractional ordinary differential equation (1.1) with right-hand side

$$
f(x, y)=-\lambda y, \quad \lambda>0 .
$$

In this case, the scheme (2.12) for $k>2$ can be rewritten as

$$
(1+\tilde{\alpha}) y_{j}=\sum_{k=0}^{j-1} d_{k}^{j} y_{k}, \quad k=3, \ldots, 2 N
$$

where

$$
\begin{aligned}
& d_{k}^{2 m+1}=-\frac{D_{k}^{(m)}}{\alpha_{0}}, \quad k=0, \ldots, 2 m, \\
& d_{k}^{2 m+2}=-\frac{\bar{D}_{k}^{(m)}}{\alpha_{0}}, \quad k=0, \ldots, 2 m+1, \\
& \alpha_{0}=D_{2 m+1}^{(m)}=\bar{D}_{2 m+2}^{(m)}=\frac{\nu+2}{\Gamma(3-\nu) 2^{\nu}}, \quad \tilde{\alpha}=\frac{\lambda \Delta x^{\nu}}{\alpha_{0}}>0 .
\end{aligned}
$$

Our purpose is to show that there exists a constant $K$ such that $\left|y_{j}\right|<K\left|y_{0}\right|$ for any $j$. Such a property would be obvious from (3.2) if all the coefficients $d_{k}^{j}$ were positive. Unfortunately, this is not true for some $\nu \in(0,1)$. The following lemma shows the properties of the coefficients $d_{k}^{j}$.

Lemma 3.1. For any $0<\nu<1, j \geq 4$, the coefficients in the scheme (3.2) satisfy

(1) $\sum_{k=0}^{j-1} d_{k}^{j}=1$.

(2) $d_{k}^{j}>\frac{2 \nu}{3 \alpha_{0} \Gamma(1-\nu)}(j-k)^{-\nu-1}, \quad k=2, \ldots, j-3$.

(3) $d_{j-1}^{j}>0, \quad d_{0}^{j}>0, \quad d_{1}^{j}>0$.

(4) There exists $\nu_{0} \in(0,1)$ such that $d_{j-2}^{j}>0$ if $\nu \in\left(0, \nu_{0}\right)$, and $d_{j-2}^{j}<0$ if $\nu \in$ $\left(\nu_{0}, 1\right)$.

(5) $\frac{1}{4}\left(d_{j-1}^{j}\right)^{2}+d_{j-2}^{j}>\frac{2^{-\nu} \nu}{8 \alpha_{0} \Gamma(1-\nu)}>0$. 
Proof. For simplicity, below we only present proof for the case $j=2 m+1, m \geq 2$. The proof for even $j$ is very similar. The statements below rely on some technical inequalities, which are provided in Appendix A.

(1) By the fact that the scheme (2.12) for $j=2 m+1$ is exact for constant solutions, we have

$$
\sum_{k=0}^{2 m} D_{k}^{(m)}+D_{2 m+1}^{(m)}=0 .
$$

According to the definition in the (3.3), we immediately obtain the equality of (1).

(2) For any $k=2, \ldots, 2 m-2$, we let $g_{k}^{(m)}=\Gamma(3-\nu) D_{k}^{(m)}$. According to (3.3), we have

$$
d_{k}^{2 m+1}=-\frac{1}{\alpha_{0} \Gamma(3-\nu)} g_{k}^{(m)},
$$

and we are going to prove

$$
g_{k}^{(m)}<-(2-\nu)(1-\nu) \nu(2 m+1-k)^{-\nu-1}
$$

by considering the following three cases separately.

Case 1: $k=2$. In this case, we claim that

$$
\begin{aligned}
g_{2}^{(m)}= & \frac{2-\nu}{2}\left[4(2 m-2)^{1-\nu}+3(2 m)^{1-\nu}-(2 m+1)^{1-\nu}\right] \\
& +\left[2(2 m-2)^{2-\nu}-3(2 m)^{2-\nu}+(2 m+1)^{2-\nu}\right] \\
\leq & -\frac{5}{4}(2-\nu)(1-\nu) \nu(2 m-1)^{-\nu-1} .
\end{aligned}
$$

To show this, we rewrite the above inequality by applying binomial expansion on both sides:

$$
\begin{aligned}
& \sum_{j=0}^{+\infty}(-1)^{j}\left(\begin{array}{l}
2-\nu \\
j+3
\end{array}\right) \frac{j+1}{2}\left(2^{j+4}-(-1)^{j}\right)\left(\frac{1}{2 m}\right)^{j+1+\nu} \\
\leq & \sum_{j=0}^{+\infty}(-1)^{j}\left(\begin{array}{l}
2-\nu \\
j+3
\end{array}\right) \frac{5}{4}(j+3)(j+2)(j+1)\left(\frac{1}{2 m}\right)^{j+1+\nu} .
\end{aligned}
$$

This inequality holds if

$$
2^{j+4}-(-1)^{j} \geq \frac{5}{2}(j+3)(j+2), \quad \forall j=0,1, \ldots .
$$

When $j=0$, this can be directly verified. When $j \geq 1$, let

$$
h(x)=2^{x+4}-1-\frac{5}{2}(x+3)(x+2) .
$$


It can be easily verified that $h(x)$ is convex when $x \geq 1$. Using $h^{\prime}(1)>0$ and $h(1)>0$, we conclude that $h(x)$ is positive for all $x \geq 1$. Therefore (3.5) holds.

Case 2: $k=4,6, \ldots, 2 m-2$. In this case,

$$
g_{k}^{(m)}=2 \tilde{g}_{k^{\prime}}^{(m)}
$$

where $k^{\prime}=m-\frac{k}{2}$ and

$$
\tilde{g}_{k^{\prime}}^{(m)}=(2-\nu)\left[\left(2 k^{\prime}\right)^{1-\nu}+\left(2 k^{\prime}+2\right)^{1-\nu}\right]+\left(2 k^{\prime}\right)^{2-\nu}-\left(2 k^{\prime}+2\right)^{2-\nu} .
$$

If $k^{\prime}>1$, by Lemma A.2, we can obtain

$$
\begin{aligned}
\tilde{g}_{k^{\prime}}^{(m)} & <-(2-\nu)\left(2 k^{\prime}\right)^{1-\nu}\left(\frac{2}{2 k^{\prime}}\right)^{2} \frac{(1-\nu) \nu}{6}\left(1-\frac{\nu+1}{2} \frac{2}{2 k^{\prime}}\right) \\
& \leq(3-\nu)\left(\begin{array}{c}
2-\nu \\
3
\end{array}\right)\left(2 k^{\prime}\right)^{-\nu-1} .
\end{aligned}
$$

Thus

$$
\begin{aligned}
g_{k}^{(m)} & <2(3-\nu)\left(\begin{array}{c}
2-\nu \\
3
\end{array}\right)(2 m-k)^{-\nu-1} \\
& <-\frac{2}{3}(2-\nu)(1-\nu) \nu(2 m+1-k)^{-\nu-1} .
\end{aligned}
$$

When $k^{\prime}=1$, by Lemma A.1(7), we get

$$
\begin{aligned}
\tilde{g}_{k^{\prime}}^{(m)} & =2^{1-\nu}\left[4-\nu-(2+\nu) 2^{1-\nu}\right] \\
& <\frac{1}{27}(2 \nu-3)(2-\nu)(1-\nu) \nu \\
& <-3^{-\nu-2}(2-\nu)(1-\nu) \nu,
\end{aligned}
$$

where we have used $3^{-\nu}<\frac{(3-2 \nu)}{3}$, which comes from the convexity of the function $3^{-\nu}$. The above inequality implies that (3.4) also holds for $k=2 m-2$.

Case 3: $k=3,5, \ldots, 2 m-3$. In this case, we have

$$
g_{k}^{(m)}=\frac{1}{2} \hat{g}_{\bar{k}}^{(m)}
$$

where $\bar{k}=m-\frac{(k-1)}{2}$, and

$$
\begin{aligned}
\hat{g}_{\bar{k}}^{(m)}= & -(2-\nu)(2 \bar{k})^{1-\nu}\left[\left(1-\frac{1}{\bar{k}}\right)^{1-\nu}+6+\left(1+\frac{1}{\bar{k}}\right)^{1-\nu}\right] \\
& -2(2 \bar{k})^{2-\nu}\left[\left(1-\frac{1}{\bar{k}}\right)^{2-\nu}-\left(1+\frac{1}{\bar{k}}\right)^{2-\nu}\right] .
\end{aligned}
$$


Since $\bar{k} \geq 2$, we can apply Lemma A.1(1),(2) to get

$$
\begin{aligned}
\hat{g}_{\bar{k}}^{(m)} \leq & -(2-\nu)(2 \bar{k})^{1-\nu}\left(8-\frac{1-\nu}{2 \bar{k}^{2}}\left[2^{\nu}-\left(\frac{2}{3}\right)^{\nu}\right]\right) \\
& -2(2 \bar{k})^{2-\nu}\left[-2(2-\nu) \frac{1}{\bar{k}}+\frac{(2-\nu)(1-\nu) \nu}{3 \bar{k}^{3}}\right] \\
= & -(2 \bar{k})^{1-\nu} \frac{(2-\nu)(1-\nu)}{6 \bar{k}^{2}}\left(8 \nu-3\left[2^{\nu}-\left(\frac{2}{3}\right)^{\nu}\right]\right) .
\end{aligned}
$$

Let

$$
f(\nu):=8 \nu-3\left[2^{\nu}-\left(\frac{2}{3}\right)^{\nu}\right]
$$

Then

$$
f^{\prime \prime}(\nu)=3\left[\left(\frac{2}{3}\right)^{\nu}\left(\log \frac{2}{3}\right)^{2}-2^{\nu}(\log 2)^{2}\right]<0 .
$$

Therefore $f(\nu) \geq f(0)(1-\nu)+f(1) \nu=4 \nu$. Thus

$$
\begin{aligned}
g_{k}^{(m)} & =\frac{1}{2} \hat{g}_{\bar{k}}^{(m)} \leq-\frac{4}{3}(2-\nu)(1-\nu) \nu(2 \bar{k})^{-1-\nu} \\
& =-\frac{4}{3}(2-\nu)(1-\nu) \nu(2 m+1-k)^{-1-\nu} .
\end{aligned}
$$

(3) All the three inequalities can be directly shown as follows:

$$
\begin{aligned}
& d_{2 m}^{2 m+1}=-\frac{D_{2 m}^{(m)}}{\alpha_{0}}=\frac{2 \nu 2^{1-\nu}}{\Gamma(3-\nu) \alpha_{0}}=\frac{4 \nu}{\nu+2}>0 \\
& d_{1}^{2 m+1}=-\frac{1}{\alpha_{0} \Gamma(3-\nu)}\left(-\frac{2-\nu}{2}\left[(2 m-2)^{1-\nu}+3(2 m)^{1-\nu}-4(2 m+1)^{1-\nu}\right]\right. \\
&\left.\quad-\left[(2 m-2)^{2-\nu}-3(2 m)^{2-\nu}+2(2 m+1)^{2-\nu}\right]\right)>0
\end{aligned}
$$

[Due to Lemma A.1(3)]

$d_{0}^{2 m+1}=-\frac{1}{\alpha_{0} \Gamma(3-\nu)}\left(\frac{2-\nu}{2}\left[(2 m)^{1-\nu}-3(2 m+1)^{1-\nu}\right]-(2 m)^{2-\nu}+(2 m+1)^{2-\nu}\right)>0$.

[Due to (A.6)]

(4) Since

$$
d_{2 m-1}^{2 m+1}=-\frac{D_{2 m-1}^{(m)}}{\alpha_{0}}=\frac{2^{1-\nu}}{\Gamma(3-\nu) \alpha_{0}}\left[3(2-\nu)-(6+\nu) 2^{-\nu}\right],
$$

the sign of $d_{2 m-1}^{2 m+1}$ is determined by the sign of $h(\nu):=3(2-\nu)-(6+\nu) 2^{-\nu}$, which satisfies

$$
h^{\prime \prime}(\nu)=-2^{-\nu}(\log 2)^{2}\left(6-\frac{2}{\log 2}+\nu\right)<0,
$$




$$
h^{\prime}(0)=6 \log 2-4>0, \quad h^{\prime}(1)=\frac{7}{2}(\log 2-1)<0 .
$$

Therefore $h(\nu)$ first increases and then decreases. By $h(0)=0$ and $h(1)=-\frac{1}{2}$, we know that $h(\nu)$ has only one zero $\nu_{0}$ in $(0,1)$, and $h(\nu)>0$ if $\nu \in\left(0, \nu_{0}\right)$ and $h(\nu)<0$ if $\nu \in\left(\nu_{0}, 1\right)$, which agrees with the conclusion of the lemma.

(5) By Lemma A.1(8), we directly have

$$
\begin{aligned}
\frac{1}{4}\left(d_{2 m}^{2 m+1}\right)^{2}+d_{2 m-1}^{2 m+1} & =\frac{2}{(2+\nu)^{2}}\left[12-\nu^{2}-\left(12+8 \nu+\nu^{2}\right) 2^{-\nu}\right] \\
& >\frac{(2-\nu)(1-\nu) \nu}{8(2+\nu)}=\frac{2^{-\nu} \nu}{8 \alpha_{0} \Gamma(1-\nu)},
\end{aligned}
$$

which completes the proof.

The above lemma shows that for $\nu<\nu_{0}$, all the coefficients $d_{k}^{j}$ are positive. In this case, as mentioned previously, the stability of the scheme can be immediately obtained from (3.2). However, this does not hold when $\nu>\nu_{0}$. To deal with this case, below we are going to rewrite the scheme (3.2) as equations with all positive coefficients. To this end, we introduce

$$
\bar{y}_{j}=y_{j}-\theta y_{j-1} \quad \text { for all } j \geqslant 1, \quad \bar{y}_{0}=y_{0},
$$

where $\theta=\frac{2 \nu}{2+\nu}$. In fact, we have $\theta=\frac{1}{2} d_{j-1}^{j}$ for all $j \geq 4$. Thus the numerical solution $y_{j}$ can be represented by $\bar{y}_{j}$ through

$$
y_{j}=\bar{y}_{j}+\theta y_{j-1}=\bar{y}_{j}+\theta \bar{y}_{j-1}+\theta^{2} y_{j-2}=\cdots=\sum_{k=0}^{j} \theta^{j-k} \bar{y}_{k} .
$$

For $j \geq 4$, we can rewrite the scheme (3.2) by subtracting both sides by $\theta y_{j-1}$ :

$$
\bar{y}_{j}+\tilde{\alpha} y_{j}=\theta y_{j-1}+\sum_{k=0}^{j-2} d_{k}^{j} y_{k}=\theta \bar{y}_{j-1}+\sum_{k=0}^{j-2}\left(\theta^{j-k}+\sum_{k^{\prime}=k}^{j-2} d_{k^{\prime}}^{j} \theta^{k^{\prime}-k}\right) \bar{y}_{k},
$$

where we have inserted (3.6) to write the right-hand side as functions of $\bar{y}_{j}$. By defining

$$
\bar{d}_{j}^{j}=-1, \quad \bar{d}_{k}^{j}=\theta^{j-k}+\sum_{k^{\prime}=k}^{j-2} d_{k^{\prime}}^{j} \theta^{k^{\prime}-k}, \quad k=0, \ldots, j-1, \quad j \geq 4,
$$

Eq. (3.7) becomes

$$
\bar{y}_{j}+\tilde{\alpha} y_{j}=\theta \bar{y}_{j-1}+\sum_{k=0}^{j-2} \bar{d}_{k}^{j} \bar{y}_{k}, \quad j \geq 4 .
$$

Note that the same equation does not hold for $j=3$. When $j=3$, we can use the same method to rewrite $\bar{y}_{3}+\tilde{\alpha} y_{3}$ as a linear combination of $\bar{y}_{0}, \bar{y}_{1}$ and $\bar{y}_{2}$. The result is

$$
\bar{y}_{3}+\tilde{\alpha} y_{3}=\bar{d}_{2}^{3} \bar{y}_{2}+\bar{d}_{1}^{3} \bar{y}_{1}+\bar{d}_{0}^{3} \bar{y}_{0},
$$


where

$$
\begin{aligned}
& \vec{d}_{2}^{3}=d_{2}^{3}-\theta=\frac{\nu+6}{\nu+2}-\frac{4+\nu}{\nu+2}\left(\frac{2}{3}\right)^{\nu-1} \\
& \vec{d}_{1}^{3}=\vec{d}_{2}^{3} \theta+d_{1}^{3}=\frac{1}{(2+\nu)^{2}}\left[-\nu^{2}-12+3\left(\nu^{2}+2 \nu+4\right)\left(\frac{2}{3}\right)^{\nu}\right], \\
& \vec{d}_{0}^{3}=\vec{d}_{1}^{3} \theta+d_{0}^{3}=\frac{1}{2} \frac{(\nu-2)^{2}}{(\nu+2)^{3}}\left[4-2 \nu+3 \nu\left(\frac{2}{3}\right)^{\nu}\right] .
\end{aligned}
$$

Additionally, we define $\bar{d}_{3}^{3}=-1$, so that for any $j \geq 3$, we have

$$
d_{k}^{j}=\bar{d}_{k}^{j}-\theta \bar{d}_{k+1}^{j}, \quad \text { for all } \quad k=0, \ldots, j-1 .
$$

The following lemma shows that in the new "scheme" (3.9), all the coefficients are positive.

Lemma 3.2. For $0<\nu<1$, the coefficients defined in (3.8) satisfy

(1) $0<\overline{d_{2}^{3}}<\theta<\frac{2}{3}$;

(2) $\bar{d}_{k}^{j}>0, \quad k=0, \ldots, j-2, \quad j \geq 3$;

(3) $\theta+\sum_{k=0}^{j-2} \bar{d}_{k}^{j}<1, \quad j \geq 3$.

Proof. (1) Using the fact that $\left(\frac{2}{3}\right)^{\nu-1}$ is a convex function, we have $\left(\frac{2}{3}\right)^{\nu-1}<\frac{3-\nu}{2}$. Therefore by (3.11),

$$
\vec{d}_{2}^{3}>\frac{\nu+6}{\nu+2}-\frac{4+\nu}{\nu+2} \frac{3-\nu}{2}=\frac{\nu(\nu+3)}{2(\nu+2)}>0 .
$$

The inequality $\bar{d}_{2}^{3}<\theta$ is a direct result of Lemma A.1(5) since

$$
\vec{d}_{2}^{3}-\theta=\frac{1}{2+\nu}\left[6-\nu-\left(2+\frac{\nu}{2}\right) 2^{\nu} 3^{1-\nu}\right] .
$$

The fact that $\theta<\frac{2}{3}$ is obvious since $\theta=\frac{2 \nu}{\nu+2}$.

(2) When $j=3$, by Lemma A.1(4) and (3.12), we immediately see that $\bar{d}_{1}^{3}>0$. The fact that $\bar{d}_{0}^{3}>0$ can be observed from

$$
\vec{d}_{0}^{3}=\frac{1}{2} \frac{(\nu-2)^{2}}{(\nu+2)^{3}}\left[4-2 \nu+3 \nu\left(\frac{2}{3}\right)^{\nu}\right]>\frac{1}{2} \frac{(\nu-2)^{2}}{(\nu+2)^{3}}(4-2 \nu+2 \nu)>0 .
$$

When $j>3$, by (3.8) and Lemma 3.1(5), we get

$$
\bar{d}_{j-2}^{j}=\theta^{2}+d_{j-2}^{j}=\frac{1}{4}\left(d_{j-1}^{j}\right)^{2}+d_{j-2}^{j}>0 .
$$


For other cases, we notice that (3.8) implies the following recurrence relation of $\bar{d}_{k}^{j}$ :

$$
\bar{d}_{k}^{j}=\theta \bar{d}_{k+1}^{j}+d_{k}^{j}, \quad k=0, \ldots, j-3 .
$$

Since $d_{k}^{j}>0$ for all $k=0, \ldots, j-3$, Eq. (3.16) shows that $\bar{d}_{k+1}^{j}>0$ implies $\bar{d}_{k}^{j}>0$. Thus, by mathematical induction with the base case (3.15), we see that $\bar{d}_{k}^{j}>0$ for all $k=0, \ldots, j-2$.

(3) When $j=3$, direct calculation yields

$$
\begin{aligned}
& \theta+\vec{d}_{1}^{3}+\vec{d}_{0}^{3} \\
= & 1+\frac{1}{2(2+\nu)^{3}}\left[-2 \nu^{3}+12 \nu^{2}-56 \nu-48+3\left(\frac{2}{3}\right)^{\nu}\left(3 \nu^{3}+4 \nu^{2}+20 \nu+16\right)\right] .
\end{aligned}
$$

By Lemma A.1(6), we see that the above quantity is less than 1 . When $j \geq 4$, we let $Q_{j}$ be the left-hand side of the inequality. It can be observed from (3.8) that

$$
(1-\theta) Q_{j}=\theta\left(1-\theta^{j}\right)+\sum_{k=0}^{j-2}\left(1-\theta^{k+1}\right) d_{k}^{j}
$$

According to Lemma 3.1(2), we have

$$
\begin{aligned}
(1-\theta) Q_{j} & <\theta\left(1-\theta^{j}\right)+\sum_{k=0}^{j-3} d_{k}^{j}+\left(1-\theta^{j-1}\right) d_{j-2}^{j} \\
& =\theta+\sum_{k=0}^{j-2} d_{k}^{j}-\theta^{j-1}\left(\theta^{2}+d_{j-2}^{j}\right),
\end{aligned}
$$

where we have used (3.15) at the last step. Now we apply Lemma 3.1(1) to get

$$
(1-\theta) Q_{j}<\theta+1-d_{j-1}^{j}=\theta+1-2 \theta=1-\theta,
$$

which indicates $Q_{j}<1$.

Base on this lemma, we can show the stability for the numerical solution $\bar{y}_{j}$.

Lemma 3.3. For $0<\nu<1$, we have

$$
\bar{y}_{j}^{2}+\tilde{\alpha} y_{j}^{2} \leq y_{0}^{2} \quad \text { for all } \quad j>0 .
$$

Proof. We first prove (3.17) for $j=1$. When $f(x, y)=-\lambda y$, the scheme (2.12) for the first two steps is

$$
\begin{aligned}
& \widehat{D}_{0} y_{0}+\widehat{D}_{1} y_{1}+\widehat{D}_{2} y_{2}=-\beta_{0} y_{1}, \\
& \widetilde{D}_{0} y_{0}+\widetilde{D}_{1} y_{1}+\widetilde{D}_{2} y_{2}=-\beta_{0} y_{2},
\end{aligned}
$$


where $\beta_{0}=\lambda \Delta x^{\nu}$. By solving the linear system, we can get

$$
\begin{aligned}
& \bar{y}_{1}^{2}+\tilde{\alpha} y_{1}^{2} \\
= & y_{0}^{2} \cdot \frac{\tilde{a}_{0}+\tilde{a}_{1} \Gamma(3-\nu) \beta_{0}+\tilde{a}_{2} \Gamma(3-\nu)^{2} \beta_{0}^{2}+\tilde{a}_{3} \Gamma(3-\nu)^{3} \beta_{0}^{3}+\tilde{a}_{4} \Gamma(3-\nu)^{4} \beta_{0}^{4}}{\tilde{b}_{0}+\tilde{b}_{1} \Gamma(3-\nu) \beta_{0}+\tilde{b}_{2} \Gamma(3-\nu)^{2} \beta_{0}^{2}+\tilde{b}_{3} \Gamma(3-\nu)^{3} \beta_{0}^{3}+\tilde{b}_{4} \Gamma(3-\nu)^{4} \beta_{0}^{4}},
\end{aligned}
$$

where the coefficients satisfy

$$
\begin{aligned}
& \tilde{a}_{0}=16(2-\nu)^{4}>0 \text {, } \\
& \tilde{a}_{1}=8(\nu-2)^{2}\left[2^{\nu}\left(5 \nu^{2}-8 \nu+12\right)-4\left(\nu^{2}+2 \nu\right)\right] \\
& \geq 8(\nu-2)^{2}\left[(1+\nu \log 2)\left(5 \nu^{2}-8 \nu+12\right)-4\left(\nu^{2}+2 \nu\right)\right]>0, \\
& \tilde{a}_{2}=16 \nu^{2}(\nu+2)^{2}-2^{\nu+3} \nu\left(5 \nu^{3}+4 \nu^{2}-28 \nu+32\right) \\
& +4^{\nu}\left(25 \nu^{4}-76 \nu^{3}+148 \nu^{2}-256 \nu+192\right) \\
& \geq 16 \nu^{2}(\nu+2)^{2}-8(1+\nu) \nu\left(5 \nu^{3}+4 \nu^{2}-28 \nu+32\right) \\
& +(1+\nu \log 4)\left(25 \nu^{4}-76 \nu^{3}+148 \nu^{2}-256 \nu+192\right)>0, \\
& \tilde{a}_{3}=2^{\nu}\left[32 \nu^{2}(\nu+2)-2^{\nu+3} \nu\left(5 \nu^{2}-10 \nu+8\right)+4^{\nu}(\nu+2)(4-3 \nu)^{2}\right] \\
& \geq 2^{\nu}\left[32 \nu^{2}(\nu+2)-8(1+\nu) \nu\left(5 \nu^{2}-10 \nu+8\right)+(1+\nu \log 4)(\nu+2)(4-3 \nu)^{2}\right]>0, \\
& \tilde{a}_{4}=4^{\nu+2} \nu^{2}>0 \text {, } \\
& \tilde{b}_{0}-\tilde{a}_{0}=128 \nu(2-\nu)^{2}>0 \text {, } \\
& \tilde{b}_{1}-\tilde{a}_{1}=8(2-\nu)\left[2^{\nu}\left(\nu^{3}-30 \nu^{2}+28 \nu-8\right)-2(\nu+2)\left(\nu^{2}-8 \nu-4\right)\right] \\
& \geq 8(2-\nu) 2^{\nu}\left[\left(\nu^{3}-30 \nu^{2}+28 \nu-8\right)-2\left(\nu^{2}-8 \nu-4\right)\right]>0, \\
& \tilde{b}_{2}-\tilde{a}_{2}=4^{\nu}\left(-9 \nu^{4}+108 \nu^{3}-196 \nu^{2}+192 \nu-128\right) \\
& +2^{\nu+3}\left(3 \nu^{4}-8 \nu^{3}-44 \nu^{2}+48 \nu+32\right)-4(\nu-2)(3 \nu+2)(\nu+2)^{2} \\
& \geq(1+\nu \log 4)\left(-9 \nu^{4}+108 \nu^{3}-196 \nu^{2}+192 \nu-128\right) \\
& +8\left(3 \nu^{4}-8 \nu^{3}-44 \nu^{2}+48 \nu+32\right)-4(\nu-2)(3 \nu+2)(\nu+2)^{2}>0, \\
& \tilde{b}_{3}-\tilde{a}_{3}=2^{\nu}\left[2^{\nu+3}\left(3 \nu^{3}-16 \nu^{2}+8 \nu+8\right)-4^{\nu}(\nu+2)(4-3 \nu)^{2}\right. \\
& -8(\nu-2)(\nu+2)(3 \nu+2)] \\
& >2^{\nu}\left[8\left(3 \nu^{3}-16 \nu^{2}+8 \nu+8\right)-4(\nu+2)(4-3 \nu)^{2}\right. \\
& -8(\nu-2)(\nu+2)(3 \nu+2)]>0, \\
& \tilde{b}_{4}-\tilde{a}_{4}=4^{1+\nu}\left(4+4 \nu-3 \nu^{2}\right)>0 \text {. }
\end{aligned}
$$

In the above derivation, we have omitted the details on the determination of signs for all polynomials of degree less than or equal to 5 , which is elementary but tedious. Since $\beta_{0}=\lambda \Delta x^{\nu}>0$, these inequalities show that the coefficient of $y_{0}^{2}$ on the right-hand side of (3.19) is less than 1 . Therefore $\bar{y}_{1}^{2}+\tilde{\alpha} y_{1}^{2} \leq y_{0}^{2}$. 
To show (3.17) for $j=2$, we also solve the linear system (3.18) to get

$$
\begin{aligned}
& \bar{y}_{2}^{2}+\tilde{\alpha} y_{2}^{2} \\
= & y_{0}^{2} \cdot \frac{\check{a}_{0}+\check{a}_{1} \Gamma(3-\nu) \beta_{0}+\check{a}_{2} \Gamma(3-\nu)^{2} \beta_{0}^{2}+\check{a}_{3} \Gamma(3-\nu)^{3} \beta_{0}^{3}}{\check{b}_{0}+\check{b}_{1} \Gamma(3-\nu) \beta_{0}+\check{b}_{2} \Gamma(3-\nu)^{2} \beta_{0}^{2}+\check{b}_{3} \Gamma(3-\nu)^{3} \beta_{0}^{3}+\check{b}_{4} \Gamma(3-\nu)^{4} \beta_{0}^{4}},
\end{aligned}
$$

and it can be similarly shown that

$$
\check{b}_{l}>\check{a}_{l}>0, \quad l=0,1,2,3, \quad \check{b}_{4}>0 .
$$

Therefore (3.17) also holds for $j=2$.

Next, we prove (3.17) for $j=3$. Multiplying by $2 \bar{y}_{3}$ on both sides of (3.10), and using the identity

$$
2 y_{j} \bar{y}_{j}=\left(y_{j}+y_{j}\right) \bar{y}_{j}=\left(y_{j}+\bar{y}_{j}+\theta y_{j-1}\right)\left(y_{j}-\theta y_{j-1}\right)=y_{j}^{2}+\bar{y}_{j}^{2}-\theta^{2} y_{j-1}^{2},
$$

we get

$$
2 \bar{y}_{3}^{2}+\tilde{\alpha} y_{3}^{2}+\tilde{\alpha} \bar{y}_{3}^{2}-\tilde{\alpha} \theta^{2} y_{2}^{2} \leq \bar{d}_{2}^{3} \bar{y}_{2}^{2}+\bar{d}_{1}^{3} \bar{y}_{1}^{2}+\bar{d}_{0}^{3} y_{0}^{2}+\left(\vec{d}_{2}^{3}+\bar{d}_{1}^{3}+\bar{d}_{0}^{3}\right) \bar{y}_{3}^{2} .
$$

Applying Lemma 3.2(1)(3) and the result (3.17) for $j=1,2$ to the above inequality, we obtain

$$
\bar{y}_{3}^{2}+\tilde{\alpha} y_{3}^{2} \leq \theta\left(\bar{y}_{2}^{2}+\tilde{\alpha} y_{2}^{2}\right)+\bar{d}_{1}^{3}\left(\bar{y}_{1}^{2}+\tilde{\alpha} y_{1}^{2}\right)+\vec{d}_{0}^{3} y_{0}^{2} \leq\left(\theta+\vec{d}_{1}^{3}+\bar{d}_{0}^{3}\right) y_{0}^{2} \leq y_{0}^{2} .
$$

Therefore, we can obtain (3.17) for $j=3$.

When $j \geq 4$, we apply mathematical induction and assume that the result holds for all cases up to $j-1$. To show (3.17), we multiply both sides of (3.9) by $2 \bar{y}_{j}$ and apply the identity (3.20), resulting in the following inequality:

$$
\begin{aligned}
& 2 \bar{y}_{j}^{2}+\tilde{\alpha} y_{j}^{2}+\tilde{\alpha} \bar{y}_{j}^{2}-\tilde{\alpha} \theta^{2} y_{j-1}^{2} \\
\leq & \theta \bar{y}_{j-1}^{2}+\sum_{k=0}^{j-2} \bar{d}_{k}^{j} \bar{y}_{k}^{2}+\left(\theta+\sum_{k=0}^{j-2} \bar{d}_{k}^{j}\right) \bar{y}_{j}^{2} \leq \theta \bar{y}_{j-1}^{2}+\sum_{k=0}^{j-2} \bar{d}_{k}^{j} \bar{y}_{k}^{2}+\bar{y}_{j}^{2},
\end{aligned}
$$

where Lemma 3.2(3) has been applied at the last step. Some rearrangement yields

$$
\bar{y}_{j}^{2}+\tilde{\alpha} y_{j}^{2} \leq \theta\left(\bar{y}_{j-1}^{2}+\tilde{\alpha} y_{j-1}^{2}\right)+\sum_{k=1}^{j-2} \bar{d}_{k}^{j}\left(\bar{y}_{k}^{2}+\tilde{\alpha} y_{k}^{2}\right)+\bar{d}_{0}^{j} y_{0}^{2} .
$$

Now one can apply the inductive hypothesis to get

$$
\bar{y}_{j}^{2}+\tilde{\alpha} y_{j}^{2} \leq\left(\theta+\sum_{k=1}^{j-2} \bar{d}_{k}^{j}+\bar{d}_{0}^{j}\right) y_{0}^{2} \leq y_{0}^{2} .
$$

By the principle of mathematical induction, the inequality (3.17) holds for all $j>0$.

By now, we are ready to show the stability of the original numerical solution $y_{k}$. 
Theorem 3.1. The scheme (2.12) for Eq. (1.1) with $f$ given in (3.1) is stable in the sense that

$$
\left|y_{k}\right| \leq \frac{2+\nu}{2-\nu}\left|y_{0}\right| \text { for all } \quad k>0 .
$$

Proof. By Lemma 3.3, we can get

$$
\left|\bar{y}_{j}\right| \leq\left|y_{0}\right| \text { for all } j>0 .
$$

Inserting this inequality to (3.6) yields

$$
\left|y_{k}\right| \leq \sum_{j=0}^{k} \theta^{k-j}\left|\bar{y}_{j}\right| \leq \sum_{j=0}^{k} \theta^{k-j}\left|y_{0}\right| \leq \frac{1}{1-\theta}\left|y_{0}\right|=\frac{2+\nu}{2-\nu}\left|y_{0}\right|,
$$

which completes the proof of the stability.

\section{Convergence analysis}

Our convergence analysis follows the general idea of the recent work [25], which is parallel to the proof of $L^{2}$-stability of the fractional ODE (1.1) with respect to the initial data. However, our analysis has to deal with the special processing of the first two time steps and the non-positivity of the coefficients in the numerical scheme. For the sake of clarity, we decompose our analysis into the following three subsections. Before that, we make the following assumptions:

(H1) The exact solution $y \in C^{3}([0, T])$;

(H2) The right-hand side $f(x, y)$ is Lipschitz continuous with respect to $y$, i.e.

$$
\left|f\left(x, y^{*}\right)-f\left(x, y^{* *}\right)\right|<L\left|y^{*}-y^{* *}\right| \quad \text { for any } y^{*} \text { and } y^{* *} .
$$

In the following analysis, we will restrict ourselves to the numerical solution exactly on $[0, T]$. As in the beginning of the Section 2, we suppose $2 N \Delta x=T$ for a positive integer $N$. For convenience, we define the numerical error by

$$
e_{j}=y\left(x_{j}\right)-y_{j}, \quad j=0, \ldots, 2 N,
$$

and $e_{0}=0$. Furthermore, by the hypotheses (H2), we can find $L_{j}$ for $j=1, \ldots, 2 N$, such that

$$
f\left(x_{j}, y\left(x_{j}\right)\right)-f\left(x_{j}, y_{j}\right)=L_{j}\left(y\left(x_{j}\right)-y_{j}\right), \quad\left|L_{j}\right| \leq L .
$$

Remark 4.1. In (H2), we have assumed the global Lipschitz continuity for the righthand side. If $f(x, y)$ is local Lipschitz continuous with respect to $y$, then the same numerical order can still be proved if the numerical solution converges. We just need 
to set sufficiently small time steps to make sure that $y_{j}$ is sufficiently close to $y\left(x_{j}\right)$ so that the complete numerical solution is within the interval

$$
I:=\left[\min _{x \in[0, T]} y(x)-\epsilon, \max _{x \in[0, T]} y(x)+\epsilon\right]
$$

for some constant $\epsilon$. Then we can set $L$ to be the maximum Lipschitz coefficient for $y \in I$, and thus the proof in this section can still be applied.

\subsection{Reformulation of the numerical scheme}

Our first step is to rewrite our numerical scheme to better match the form of the Caputo derivative (1.2). To this end, we introduce the notation

$$
\nabla \psi_{k}=\psi_{k}-\psi_{k-1}, \quad k>0
$$

for any quantity $\psi_{k}$, as corresponds to the first-order derivative appearing in the definition of the Caputo derivative. Furthermore, for any $n \geq 2$ and $k=0, \ldots, n-1$, we define $\bar{B}_{k}^{n}$ as

$$
\bar{B}_{0}^{n}=\Delta x^{-\nu} \alpha_{0}, \quad \bar{B}_{n-k}^{n}=\bar{B}_{n-k-1}^{n}-\Delta x^{-\nu} \alpha_{0} \bar{d}_{k}^{n}, \quad k=1, \ldots, n-1 .
$$

By (3.14), we have

$$
\begin{aligned}
{ }_{0} D_{\Delta x}^{\nu} y_{n} & =\Delta x^{-\nu} \alpha_{0} y_{n}-\Delta x^{-\nu} \alpha_{0} \sum_{k=0}^{n-1} d_{k}^{n} y_{k}=\Delta x^{-\nu} \alpha_{0} y_{n}-\Delta x^{-\nu} \alpha_{0} \sum_{k=0}^{n-1}\left(\bar{d}_{k}^{n}-\theta \bar{d}_{k+1}^{n}\right) y_{k} \\
& =\Delta x^{-\nu} \alpha_{0}\left(y_{n}+\theta \bar{d}_{n}^{n} y_{n-1}\right)-\Delta x^{-\nu} \alpha_{0} \sum_{k=1}^{n-1} \bar{d}_{k}^{n}\left(y_{k}-\theta y_{k-1}\right)-\Delta x^{-\nu} \alpha_{0} \bar{d}_{0}^{n} y_{0} \\
& =\Delta x^{-\nu} \alpha_{0} \bar{y}_{n}-\Delta x^{-\nu} \alpha_{0} \sum_{k=1}^{n-1} \bar{d}_{k}^{n} \bar{y}_{k}-\Delta x^{-\nu} \alpha_{0} \bar{d}_{0}^{n} y_{0} .
\end{aligned}
$$

Now we can apply the definition of $\bar{B}_{k}^{n}$ given in (4.3) to rewrite the discrete fractional derivative as

$$
\begin{aligned}
{ }_{0} D_{\Delta x}^{\nu} y_{n} & =\bar{B}_{0}^{n} \bar{y}_{n}+\sum_{k=1}^{n-1}\left(\bar{B}_{n-k}^{n}-\bar{B}_{n-k-1}^{n}\right) \bar{y}_{k}-\Delta x^{-\nu} \alpha_{0} \bar{d}_{0}^{n} y_{0} \\
& =\sum_{k=1}^{n} \bar{B}_{n-k}^{n} \nabla \bar{y}_{k}+\left(\bar{B}_{n-1}^{n}-\Delta x^{-\nu} \alpha_{0} \bar{d}_{0}^{n}\right) y_{0} .
\end{aligned}
$$

Similarly, if we define $\bar{y}(x)=y(x)-\theta y(x-\Delta x)$ and $\bar{y}(0)=y_{0}$, we have

$$
{ }_{0} D_{\Delta x}^{\nu} y\left(x_{n}\right)=\sum_{k=1}^{n} \bar{B}_{n-k}^{n} \nabla \bar{y}\left(x_{k}\right)+\left(\bar{B}_{n-1}^{n}-\Delta x^{-\nu} \alpha_{0} \bar{d}_{0}^{n}\right) y_{0} .
$$

Our analysis will be based on such a form of the discrete Caputo derivative. The following lemma provides the lower bounds and the monotonicity of the coefficients. 
Lemma 4.1. The coefficients $\bar{B}_{k}^{n}$ satisfy $\bar{B}_{0}^{n}>\ldots>\bar{B}_{n-1}^{n}>0$ and

$$
\begin{aligned}
\bar{B}_{k}^{n} & \geq \frac{1}{\pi_{B} \Delta x} \int_{x_{n-k-1}}^{x_{n-k}} \omega_{1-\nu}\left(x_{n}-s\right) d s \\
& =\frac{1}{\pi_{B} \Delta x^{\nu} \Gamma(2-\nu)}\left[(k+1)^{1-\nu}-k^{1-\nu}\right],
\end{aligned}
$$

where $\pi_{B}=9$.

Proof. The monotonicity of the coefficients $\bar{B}_{k}^{n}$ is obvious by the definition (4.3) and the positivity of $\bar{d}_{k}^{n}$, and below we focus only on the proof of (4.6).

When $k=0$, we have

$$
\bar{B}_{0}^{n}=\Delta x^{-\nu} \alpha_{0}=\frac{\nu+2}{(2-\nu) 2^{\nu}} \frac{1}{\Delta x^{\nu} \Gamma(2-\nu)} \geq \frac{1}{\Delta x^{\nu} \Gamma(2-\nu)} .
$$

When $k>0$, since $(k+1)^{1-\nu}-k^{1-\nu} \leq(1-\nu) k^{-\nu}$, we just need to show

$$
\bar{B}_{k}^{n} \geq \frac{1}{9 \Delta x^{\nu} \Gamma(2-\nu)}(1-\nu) k^{-\nu}=\frac{1}{9 \Delta x^{\nu} \Gamma(1-\nu)} k^{-\nu} .
$$

Below we separate our proof into four cases.

Case 1: $n=3$. By direct calculation, one can obtain

$$
\begin{aligned}
\bar{B}_{1}^{3} & =\bar{B}_{0}^{3}-\Delta x^{-\nu} \alpha_{0} \bar{d}_{2}^{3} \\
& =\frac{2^{-\nu}}{\Delta x^{\nu} \Gamma(3-\nu)}\left[\frac{3(\nu+4)}{2}\left(\frac{2}{3}\right)^{\nu}-4\right] \\
& \geq \frac{1-\nu \log 2}{\Delta x^{\nu} \Gamma(3-\nu)}\left[\frac{3(\nu+4)}{2}\left(1+\nu \log \frac{2}{3}\right)-4\right] \\
& =\frac{1}{\Delta x^{\nu} \Gamma(1-\nu)}\left[1+\frac{\nu\left(\beta_{0}+\beta_{1} \nu+\beta_{2} \nu^{2}\right)}{2(2-\nu)(1-\nu)}\right],
\end{aligned}
$$

where

$$
\begin{aligned}
& \beta_{0}=9-12 \log 3+8 \log 2, \\
& \beta_{1}=3 \log 3(4 \log 2-1)-12(\log 2)^{2}-2, \\
& \beta_{2}=3 \log 2(\log 3-\log 2) .
\end{aligned}
$$

It is not difficult to check that $\beta_{0}+\beta_{1} \nu+\beta_{2} \nu^{2}>0$ when $\nu \in(0,1)$. Therefore

$$
\bar{B}_{1}^{3} \geq \frac{1}{\Delta x^{\nu} \Gamma(1-\nu)}
$$


Similarly, the case $k=2$ can be shown by

$$
\begin{aligned}
\bar{B}_{2}^{3} & =\bar{B}_{1}^{3}-\Delta x^{-\nu} \alpha_{0} \bar{d}_{1}^{3} \\
& =\frac{2^{-\nu}}{\Delta x^{\nu} \Gamma(2-\nu)} \cdot \frac{1}{\nu+2}\left[2-\nu+\frac{3 \nu}{2}\left(\frac{2}{3}\right)^{\nu}\right] \\
& \geq \frac{2^{-\nu}}{\Delta x^{\nu} \Gamma(2-\nu)} \cdot \frac{1}{\nu+2}\left[2-\nu+\frac{3 \nu}{2}\left(1+\nu \log \frac{2}{3}\right)\right] \\
& =\frac{2^{-\nu}}{\Delta x^{\nu} \Gamma(1-\nu)}\left[1+\frac{\nu[3+(2-3 \log 3+3 \log 2) \nu]}{2(2+\nu)(1-\nu)}\right] \\
& \geq \frac{2^{-\nu}}{\Delta x^{\nu} \Gamma(1-\nu)} .
\end{aligned}
$$

Case 2: $n>3$ and $k=n-1$. By definition,

$$
\bar{B}_{n-1}^{n}=\bar{B}_{0}^{n}-\Delta x^{-\nu} \alpha_{0} \sum_{k=1}^{n-1} \bar{d}_{k}^{n}=\Delta x^{-\nu} \alpha_{0}\left(1-\sum_{k=1}^{n-1} \bar{d}_{k}^{n}\right) .
$$

By Lemma 3.2(3) and (3.14), we can bound $\bar{B}_{n-1}^{n}$ by

$$
\bar{B}_{n-1}^{n}>\Delta x^{-\nu} \alpha_{0} \bar{d}_{0}^{n}>\Delta x^{-\nu} \alpha_{0} d_{0}^{n} .
$$

Now we consider odd and even $n$ separately. If $n=2 m+1$ and $m>1$, by (3.3a),

$$
>\frac{1}{\Delta x^{\nu} \Gamma(3-\nu)}\left(\frac{\bar{B}_{2 m}^{2 m+1}}{2}\left[3(2 m+1)^{1-\nu}-(2 m)^{1-\nu}\right]-\left[(2 m+1)^{2-\nu}-(2 m)^{2-\nu}\right]\right) .
$$

Using the inequality (A.6), we see that

$$
\bar{B}_{2 m}^{2 m+1} \geq \frac{1}{\Delta x^{\nu} \Gamma(3-\nu)}(2-\nu)(1-\nu)(2 m)^{-\nu}\left(1-\frac{7 \nu}{24 m}\right)>\frac{1}{2 \Delta x^{\nu} \Gamma(1-\nu)}(2 m)^{-\nu} .
$$

Similarly, when $n=2 m+2$ and $m \geq 1$, we have

$$
\begin{aligned}
\bar{B}_{2 m+1}^{2 m+2} & \geq \frac{1}{\Delta x^{\nu} \Gamma(3-\nu)}(2-\nu)(1-\nu)(2 m)^{-\nu}\left(1-\frac{5 \nu}{6 m}\right) \\
& =\frac{1}{\Delta x^{\nu} \Gamma(3-\nu)}(2-\nu)(1-\nu)(2 m+1)^{-\nu}\left(\frac{2 m}{2 m+1}\right)^{-\nu}\left(1-\frac{5 \nu}{6 m}\right) \\
& >\frac{1}{6 \Delta x^{\nu} \Gamma(1-\nu)}(2 m+1)^{-\nu} .
\end{aligned}
$$

Case 3: $n>3$ and $k=n-2$. We can directly use the result for $k=n-1$ to get $\bar{B}_{n-2}^{n} \geq \bar{B}_{n-1}^{n}>\frac{1}{6 \Delta x^{\nu} \Gamma(1-\nu)}(n-2)^{-\nu} \cdot\left(\frac{n-1}{n-2}\right)^{-\nu}>\frac{1}{9 \Delta x^{\nu} \Gamma(1-\nu)}(n-2)^{-\nu}$. 
Case 4: $n>3$ and $k=1, \ldots, n-3$. Using

$$
\begin{aligned}
& \bar{d}_{j}^{n}=\theta \bar{d}_{j+1}^{n}+d_{j}^{n} \geq d_{j}^{n}>\frac{2 \nu}{3 \alpha_{0} \Gamma(1-\nu)}(n-j)^{-\nu-1}, \quad j=1, \ldots, n-3, \\
& \bar{d}_{n-2}^{n}=\frac{1}{4}\left(d_{n-1}^{n}\right)^{2}+d_{n-2}^{n}>\frac{\nu}{4 \alpha_{0} \Gamma(1-\nu)} 2^{-\nu-1},
\end{aligned}
$$

we obtain

$$
\begin{aligned}
\bar{B}_{k}^{n} & =\bar{B}_{n-1}^{n}+\Delta x^{-\nu} \alpha_{0} \sum_{j=1}^{n-1-k} \bar{d}_{j}^{n}>\bar{B}_{n-1}^{n}+\frac{\nu}{4 \Delta x^{\nu} \Gamma(1-\nu)} \sum_{j=2}^{n-1-k}(n-j)^{-\nu-1} \\
& \geq \frac{1}{2 \Delta x^{\nu} \Gamma(1-\nu)}(n-1)^{-\nu}+\frac{\nu}{4 \Delta x^{\nu} \Gamma(1-\nu)} \int_{1}^{n-1-k}(n-x)^{-\nu-1} \mathrm{~d} x \\
& =\frac{1}{2 \Delta x^{\nu} \Gamma(1-\nu)}(n-1)^{-\nu}+\frac{1}{4 \Delta x^{\nu} \Gamma(1-\nu)}\left[(1+k)^{-\nu}-(n-1)^{-\nu}\right] \\
& \geq \frac{1}{4 \Delta x^{\nu} \Gamma(1-\nu)}(1+k)^{-\nu} \geq \frac{1}{8 \Delta x^{\nu} \Gamma(1-\nu)} k^{-\nu}
\end{aligned}
$$

This completes the proof for all $k=0, \ldots, n-1$.

The purpose of the above lemma is an upper bound for the discrete fractional derivative of $\left|\bar{e}_{j}\right|^{2}$. We state the result in the following lemma:

Lemma 4.2. For any $j \geq 3$,

$$
2 \bar{e}_{j} \sum_{k=3}^{j} \bar{B}_{j-k}^{j} \nabla \bar{e}_{k} \geq \sum_{k=3}^{j} \bar{B}_{j-k}^{j} \nabla\left(\left|\bar{e}_{k}\right|^{2}\right) .
$$

We refer the readers to [25, Lemma A.1] for the proof of this lemma.

\subsection{Estimation of the truncation errors}

Most error estimation is based on the estimation of the truncation error. In our case, it can be defined by

$$
r_{j}(\Delta x):={ }_{0} D_{x}^{\nu} y\left(x_{j}\right)-{ }_{0} D_{\Delta x}^{\nu} y\left(x_{j}\right), \quad j \geq 1 .
$$

Here ${ }_{0} D_{\Delta x}^{\nu} y\left(x_{j}\right)$ is defined by replacing $y_{j}$ in (2.2)-(2.4) and (2.8) with $y\left(x_{j}\right)$. As mentioned previously, the first two time steps in our scheme have to be taken into account independently. Therefore, for $j \geq 3$ we introduce the following modified truncation error:

$$
\tilde{r}_{j}(\Delta x)=r_{j}(\Delta x)-L_{j} \sum_{k=1}^{2} \theta^{j-k} \bar{e}_{k}+\sum_{k=1}^{2} \bar{B}_{j-k}^{j} \nabla \bar{e}_{k},
$$

where $\bar{e}_{k}=e_{k}-\theta e_{k-1}$. Below we are going to derive bounds for both (4.7) and (4.8). 
Theorem 4.1. Assume that (H1) holds. There exists a constant $C_{1}$ depending only on the function $y$ and the final time $T$, such that for all $\Delta x>0$,

$$
\left|r_{j}(\Delta x)\right| \leq C_{1} \Delta x^{3-\nu}, \quad j=1, \ldots, 2 N
$$

Proof. Our error estimation will be established on the following error term of the Lagrange interpolation:

$$
\begin{aligned}
& y(x)-I_{\left[x_{k}, x_{k+2}\right]} y(x) \\
= & \frac{y^{(3)}\left(\xi_{k}(x)\right)}{6}\left(x-x_{k}\right)\left(x-x_{k+1}\right)\left(x-x_{k+2}\right), \quad \forall x \in\left[x_{k}, x_{k+2}\right],
\end{aligned}
$$

where $\xi_{k}(x)$ is a function defined on $\left[x_{k}, x_{k+2}\right]$ with range $\left(x_{k}, x_{k+2}\right)$. Let $M_{1}$ be the upper bound of $y^{(3)}$ on $[0, T]$. For any $x \in\left[x_{k}, x_{k+2}\right]$, we have

$$
\left|y(x)-I_{\left[x_{k}, x_{k+2}\right]} y(x)\right| \leq \frac{M_{1}}{3} \Delta x^{2}\left(x_{k+2}-x\right)
$$

or more simply,

$$
\left|y(x)-I_{\left[x_{k}, x_{k+2}\right]} y(x)\right| \leq \frac{M_{1}}{6} \Delta x\left(x_{k+2}-x\right)\left(x-x_{k}\right) \leq \frac{M_{1}}{6} \Delta x^{3} .
$$

We first estimate $r_{1}(\Delta x)$ :

$$
\begin{aligned}
& \left|r_{1}(\Delta x)\right| \\
= & \left|\frac{1}{\Gamma(1-\nu)} \int_{0}^{x_{1}} y^{\prime}(s)\left(x_{1}-s\right)^{-\nu} d s-\frac{1}{\Gamma(1-\nu)} \int_{0}^{x_{1}}\left[I_{\left[x_{0}, x_{2}\right]} y(s)\right]^{\prime}\left(x_{1}-s\right)^{-\nu} d s\right| \\
= & \frac{\nu}{\Gamma(1-\nu)}\left|\int_{0}^{x_{1}}\left[y(s)-I_{\left[x_{0}, x_{2}\right]} y(s)\right]\left(x_{1}-s\right)^{-\nu-1} d s\right| \\
= & \frac{\nu}{\Gamma(1-\nu)}\left|\int_{0}^{x_{1}} \frac{y^{(3)}\left(\xi_{0}(s)\right)}{6}\left(s-x_{0}\right)\left(x_{1}-s\right)^{-\nu}\left(s-x_{2}\right) d s\right| \\
\leq & \frac{\nu}{\Gamma(1-\nu)} \frac{M_{1}}{6} \int_{0}^{x_{1}} s\left(x_{2}-s\right)\left(x_{1}-s\right)^{-\nu} d s=\frac{\nu}{(3-\nu) \Gamma(2-\nu)} \frac{M_{1}}{3} \Delta x^{3-\nu} \\
< & M_{1} \Delta x^{3-\nu}
\end{aligned}
$$

where we have used $\Gamma(2-\nu)>\frac{2}{3}$. The Eq. (4.13) proves (4.9) for $j=1$. The case $j=2$ can be similarly proven, and here we omit the details.

Now we estimate $r_{2 m+1}(\Delta x)$ for $m \geq 1$. In a similar way to (4.13), we can use 
integration by parts to obtain

$$
\begin{aligned}
& \quad\left|r_{2 m+1}(\Delta x)\right| \\
& =\mid \frac{\nu}{\Gamma(1-\nu)} \int_{0}^{x_{1}}\left[y(s)-I_{\left[x_{0}, x_{2}\right]} y(s)\right]\left(x_{2 m+1}-s\right)^{-\nu-1} d s \\
& \quad+\frac{\nu}{\Gamma(1-\nu)} \sum_{k=1}^{m-1} \int_{x_{2 k-1}}^{x_{2 k+1}}\left[y(s)-I_{\left[x_{2 k-1}, x_{2 k+1}\right]} y(s)\right]\left(x_{2 m+1}-s\right)^{-\nu-1} d s \\
& \quad+\frac{\nu}{\Gamma(1-\nu)} \int_{x_{2 m-1}}^{x_{2 m+1}}\left[y(s)-I_{\left[x_{2 m-1}, x_{2 m+1}\right]} y(s)\right]\left(x_{2 m+1}-s\right)^{-\nu-1} d s \mid .
\end{aligned}
$$

Applying (4.11) and (4.12), we can estimate the truncation error by

$$
\begin{aligned}
& \left|r_{2 m+1}(\Delta x)\right| \\
\leq & \frac{\nu}{\Gamma(1-\nu)}\left[\frac{M_{1}}{6} \Delta x^{3}\left(\int_{0}^{x_{1}}\left(x_{2 m+1}-s\right)^{-\nu-1} d s+\sum_{k=1}^{m-1} \int_{x_{2 k-1}}^{x_{2 k+1}}\left(x_{2 m+1}-s\right)^{-\nu-1} d s\right)\right] \\
& +\frac{\nu}{\Gamma(1-\nu)} \int_{x_{2 m-1}}^{x_{2 m+1}} \frac{M_{1}}{3} \Delta x^{2}\left(x_{2 m+1}-s\right)^{-\nu} d s \\
\leq & \frac{M_{1} \nu}{\Gamma(1-\nu)}\left[\frac{1}{6} \Delta x^{3} \int_{0}^{x_{2 m-1}}\left(x_{2 m+1}-s\right)^{-\nu-1} d s+\frac{1}{3} \Delta x^{2} \int_{x_{2 m-1}}^{x_{2 m+1}}\left(x_{2 m+1}-s\right)^{-\nu} d s\right] \\
= & \frac{M_{1}}{6 \Gamma(2-\nu)}\left[2^{-\nu}(1+3 \nu)-(1-\nu)(2 m+1)^{-\nu}\right] \Delta x^{3-\nu} \leq M_{1} \Delta x^{3-\nu} .
\end{aligned}
$$

The case $j=2 m+2$ can be similarly proven, and the details are omitted.

To show the error bounds for (4.8), we need the error estimation for the first two time steps.

Lemma 4.3. Assume that both (H1) and (H2) hold, and $\Delta x^{\nu}<(7 L)^{-1}$. Then

$$
\left|e_{1}\right| \leqslant 10 C_{1} \Delta x^{3}, \quad\left|e_{2}\right| \leqslant 10 C_{1} \Delta x^{3},
$$

where the constant $C_{1}$ is defined in Theorem 4.1.

Proof. By the numerical scheme (2.12) for $k=1,2$ and the definition of the truncation error (4.7), we have

$$
\left\{\begin{array}{l}
\Delta x^{-\nu} \widehat{D}_{1} e_{1}+\Delta x^{-\nu} \widehat{D}_{2} e_{2}=f\left(x_{1}, y\left(x_{1}\right)\right)-f\left(x_{1}, y_{1}\right)-r_{1}(\Delta x), \\
\Delta x^{-\nu} \widetilde{D}_{1} e_{1}+\Delta x^{-\nu} \widetilde{D}_{2} e_{2}=f\left(x_{2}, y\left(x_{2}\right)\right)-f\left(x_{2}, y_{2}\right)-r_{2}(\Delta x) .
\end{array}\right.
$$


After solving the equation, we get

$$
\begin{aligned}
\left|e_{1}\right|= & \Gamma(2-\nu) \Delta x^{\nu} \mid \frac{2+\nu}{2}\left[f\left(x_{1}, y\left(x_{1}\right)\right)-f\left(x_{1}, y_{1}\right)-r_{1}(\Delta x)\right] \\
& \quad-2^{\nu-2} \nu\left[f\left(x_{2}, y\left(x_{2}\right)\right)-f\left(x_{2}, y_{2}\right)-r_{2}(\Delta x)\right] \mid \\
\leq & \Gamma(2-\nu) \Delta x^{\nu}\left(\frac{2+\nu}{2}\left(L\left|e_{1}\right|+C_{1} \Delta x^{3-\nu}\right)+2^{\nu-2} \nu\left(L\left|e_{2}\right|+C_{1} \Delta x^{3-\nu}\right)\right) \\
\leq & \Delta x^{\nu}\left(\frac{3}{2}\left(L\left|e_{1}\right|+C_{1} \Delta x^{3-\nu}\right)+\frac{1}{2}\left(L\left|e_{2}\right|+C_{1} \Delta x^{3-\nu}\right)\right) \\
\leq & \frac{3}{2} L \Delta x^{\nu}\left(\left|e_{1}\right|+\left|e_{2}\right|\right)+2 C_{1} \Delta x^{3} .
\end{aligned}
$$

By similar means, we can obtain $\left|e_{2}\right| \leq 2 L \Delta x^{\nu}\left(\left|e_{1}\right|+\left|e_{2}\right|\right)+3 C_{1} \Delta x^{3}$. Summing up the two inequalities yields

$$
\left|e_{1}\right|+\left|e_{2}\right| \leq \frac{7}{2} L \Delta x^{\nu}\left(\left|e_{1}\right|+\left|e_{2}\right|\right)+5 C_{1} \Delta x^{3} .
$$

Therefore when $\Delta x^{\nu}<(7 L)^{-1}$, we have

$$
\left|e_{1}\right|+\left|e_{2}\right| \leq 10 C_{1} \Delta x^{3}
$$

which completes the proof.

The above lemma already shows that we do not lose any numerical accuracy for the first two time steps. In fact, their orders are slightly higher than the general error bound $\mathcal{O}\left(\Delta x^{3-\nu}\right)$. This is necessary to provide error bounds for $\tilde{r}_{j}(\Delta x)$ in the following theorem.

Theorem 4.2. Assume both (H1) and (H2) hold, and $\Delta x^{\nu}<(10|L|)^{-1}$. There exists a constant $C$ such that

$$
\left|\tilde{r}_{j}(\Delta x)\right| \leq C \Delta x^{3-\nu} .
$$

Proof. We first estimate the coefficients $\bar{B}_{j-1}^{j}$ and $\bar{B}_{j-2}^{j}$. According to (4.3),

$$
\begin{aligned}
& \bar{B}_{j-1}^{j}<\Delta x^{-\nu} \alpha_{0}=\frac{(\nu+2) \Delta x^{-\nu}}{\Gamma(3-\nu) 2^{\nu}} \leq 3 \Delta x^{-\nu}, \\
& \bar{B}_{j-2}^{j}-\bar{B}_{j-1}^{j}=\Delta x^{-\nu} \alpha_{0} \bar{d}_{1}^{j}<\Delta x^{-\nu} \alpha_{0} \leq 3 \Delta x^{-\nu},
\end{aligned}
$$

where we have used $\bar{d}_{1}^{j}<1$ implied by Lemma 3.2(3),(5). Now we can apply triangle inequality to (4.8)

$$
\begin{aligned}
\left|\tilde{r}_{j}(\Delta x)\right| & \leq\left|r_{j}(\Delta x)\right|+\left|(1+\theta) \bar{B}_{j-2}^{j}-\bar{B}_{j-1}^{j}\right| \cdot\left|e_{1}\right|+\left|L_{j} \theta^{j-2}-\bar{B}_{j-2}^{j}\right| \cdot\left|e_{2}\right| \\
& \leq C_{1} \Delta x^{3-\nu}+70 C_{1} \Delta x^{3-\nu}+10 C_{1}\left(\left|L_{j}\right|+6 \Delta x^{-\nu}\right) \Delta x^{3} .
\end{aligned}
$$

Here we have applied Theorem 4.1 and Lemma 4.3. Since $\Delta x^{\nu}<(10|L|)^{-1}$, the above inequality yields

$$
\left|\tilde{r}_{j}(\Delta x)\right| \leq 132 C_{1} \Delta x^{3-\nu}
$$




\subsection{Error analysis}

Now we are ready to summarize the previous two subsections and carry out the error analysis for our scheme. The purpose of Section 4.1 is to provide preparatory works to introduce an important tool - the complementary discrete convolution kernels. Inspired by the property (1.4), we would like to find the discrete kernel $P_{j}^{n}$, corresponding to the kernel $\omega_{\nu}(\cdot)$, which satisfies

$$
\sum_{j=m}^{n} P_{n-j}^{n} \bar{B}_{j-m}^{j} \equiv 1, \quad \forall 3 \leq m \leq n \leq 2 N .
$$

According [25, Eq. (2.6)], we have

$$
P_{0}^{n}=\frac{1}{\bar{B}_{0}^{n}}, \quad P_{j}^{n}=\frac{1}{\bar{B}_{0}^{n-j}} \sum_{k=0}^{j-1}\left(\bar{B}_{j-k-1}^{n-k}-\bar{B}_{j-k}^{n-k}\right) P_{k}^{n} \quad \text { for } \quad 1 \leq j \leq n-3 .
$$

Define

$$
P_{n-2}^{n}=0, \quad P_{n-1}^{n}=0
$$

Then when $m=1,2$, we have

$$
\begin{aligned}
& \sum_{j=1}^{n} P_{n-j}^{n} \bar{B}_{j-1}^{j}=P_{n-1}^{n} \bar{B}_{0}^{j}+P_{n-2}^{n} \bar{B}_{1}^{j}+\sum_{j=3}^{n} P_{n-j}^{n} \bar{B}_{j-1}^{j} \leq \sum_{j=3}^{n} P_{n-j}^{n} \bar{B}_{j-3}^{j}=1, \\
& \sum_{j=2}^{n} P_{n-j}^{n} \bar{B}_{j-2}^{j}=P_{n-2}^{n} \bar{B}_{0}^{j}+\sum_{j=3}^{n} P_{n-j}^{n} \bar{B}_{j-2}^{j} \leq \sum_{j=3}^{n} P_{n-j}^{n} \bar{B}_{j-3}^{j}=1 .
\end{aligned}
$$

By Lemma 4.1, (4.18) and (4.19), we know that all the coefficients $P_{j}^{n} \geq 0$. These coefficients help us "invert" the discrete fractional derivative, so that we can derive the recursive inequality for the numerical error.

Lemma 4.4. For any $n \geq 3$, it holds that

$$
\left|\bar{e}_{n}\right|^{2} \leq \sum_{j=3}^{n} P_{n-j}^{n} \sum_{k=3}^{j} 4 L \theta^{j-k}\left|\bar{e}_{k}\right|^{2}+\left|\bar{e}_{2}\right|^{2}+2 \sum_{j=3}^{n} P_{n-j}^{n}\left|\bar{e}_{j}\right| \cdot\left|\tilde{r}_{j}(\Delta x)\right|,
$$

where $\tilde{r}_{j}(\Delta x)$ is defined in (4.8).

Proof. Plugging (4.5) and (1.1) into (4.7), we get

$$
\begin{aligned}
r_{j}(\Delta x) & =f\left(x_{j}, y\left(x_{j}\right)\right)-\sum_{k=1}^{j} \bar{B}_{j-k}^{j} \nabla \bar{y}\left(x_{k}\right)-\left(\bar{B}_{n-1}^{n}-\Delta x^{-\nu} \alpha_{0} \bar{d}_{0}^{n}\right) y_{0} \\
& =f\left(x_{j}, y\left(x_{j}\right)\right)-\sum_{k=1}^{j} \bar{B}_{j-k}^{j} \nabla \bar{e}_{k}-\sum_{k=1}^{j} \bar{B}_{j-k}^{j} \nabla \bar{y}_{k}-\left(\bar{B}_{n-1}^{n}-\Delta x^{-\nu} \alpha_{0} \bar{d}_{0}^{n}\right) y_{0} .
\end{aligned}
$$


By (4.4), (2.12) and (4.2), the above equation can be further simplified, namely,

$$
\begin{aligned}
r_{j}(\Delta x) & =f\left(x_{j}, y\left(x_{j}\right)\right)-f\left(x_{j}, y_{j}\right)-\sum_{k=1}^{j} \bar{B}_{j-k}^{j} \nabla \bar{e}_{k} \\
& =L_{j} e_{j}-\sum_{k=1}^{j} \bar{B}_{j-k}^{j} \nabla \bar{e}_{k}=L_{j} \sum_{k=1}^{j} \theta^{j-k} \bar{e}_{k}-\sum_{k=1}^{j} \bar{B}_{j-k}^{j} \nabla \bar{e}_{k} .
\end{aligned}
$$

Now we use (4.8) to rewrite the above equation as

$$
\sum_{k=3}^{j} \bar{B}_{j-k}^{j} \nabla \bar{e}_{k}=L_{j} \sum_{k=3}^{j} \theta^{j-k} \bar{e}_{k}-\tilde{r}_{j}(\Delta x) .
$$

Now we multiply both sides of the above equation by $2 \bar{e}_{j}$. The right-hand side can be bounded by

$$
\begin{aligned}
& 2 \bar{e}_{j}\left[L_{j} \sum_{k=3}^{j} \theta^{j-k} \bar{e}_{k}-\tilde{r}_{j}(\Delta x)\right] \\
\leq & L \sum_{k=3}^{j} \theta^{j-k}\left(\left|\bar{e}_{j}\right|^{2}+\left|\bar{e}_{k}\right|^{2}\right)+2\left|\bar{e}_{j}\right| \cdot\left|\tilde{r}_{j}(\Delta x)\right| \\
\leq & \sum_{k=3}^{j} 4 L \theta^{j-k}\left|\bar{e}_{k}\right|^{2}+2\left|\bar{e}_{j}\right| \cdot\left|\tilde{r}_{j}(\Delta x)\right|,
\end{aligned}
$$

where we have used $\theta<\frac{2}{3}$, and the left-hand side can be bounded from below by Lemma 4.2. Catenating both bounds using (4.23), we see that

$$
\sum_{k=3}^{j} 4 L \theta^{j-k}\left|\bar{e}_{k}\right|^{2}+2\left|\bar{e}_{j}\right| \cdot\left|\tilde{r}_{j}(\Delta x)\right| \geq \sum_{k=3}^{j} \bar{B}_{j-k}^{j} \nabla\left(\left|\bar{e}_{k}\right|^{2}\right) .
$$

Multiplying both sides of the above equation by $P_{n-j}^{j}$ and taking the sum over $j$, one gets

$$
\begin{aligned}
& \sum_{j=3}^{n} P_{n-j}^{j} \sum_{k=3}^{j} 4 L \theta^{j-k}\left|\bar{e}_{k}\right|^{2}+2 \sum_{j=3}^{n} P_{n-j}^{j}\left|\bar{e}_{j}\right| \cdot\left|\tilde{r}_{j}(\Delta x)\right| \\
\geq & \sum_{j=3}^{n} P_{n-j}^{j} \sum_{k=3}^{j} \bar{B}_{j-k}^{j} \nabla\left(\left|\bar{e}_{k}\right|^{2}\right) .
\end{aligned}
$$

Applying the identity (4.17) yields

$$
\begin{aligned}
& \sum_{j=3}^{n} P_{n-j}^{j} \sum_{k=3}^{j} 4 L \theta^{j-k}\left|\bar{e}_{k}\right|^{2}+2 \sum_{j=3}^{n} P_{n-j}^{j}\left|\bar{e}_{j}\right| \cdot\left|\tilde{r}_{j}(\Delta x)\right| \\
\geq & \sum_{k=3}^{n} \nabla\left(\left|\bar{e}_{k}\right|^{2}\right)=\left|\bar{e}_{n}\right|^{2}-\left|\bar{e}_{2}\right|^{2},
\end{aligned}
$$


which is clearly equivalent to the conclusion of the Lemma (4.22).

We can now apply mathematical induction to bound the error by the initial error and the truncation errors.

Lemma 4.5. Let $\bar{e}_{n}=e_{n}-\theta e_{n-1}$ with $\theta=\frac{2 \nu}{(2+\nu)}$. If

$$
\Delta x^{\nu} \leq \frac{1}{24 \pi_{B} L}
$$

it holds that

$$
\left|\bar{e}_{n}\right| \leq 2 E_{\nu}\left(24 \pi_{B} L x_{n}^{\nu}\right)\left(\left|\bar{e}_{2}\right|+2 \max _{3 \leq k \leq n} \sum_{j=3}^{k} P_{k-j}^{k}\left|\tilde{r}_{j}(\Delta x)\right|\right), \quad \text { for } \quad n \geq 2,
$$

where $\tilde{r}_{j}(\Delta x)$ is defined in (4.8), and $E_{\nu}$ is the Mittag-Leffler function defined by (B.8).

Proof. In the following proof, we need some useful properties of the kernel $P_{j}^{n}$ provided in Appendix B, wherein the complete details can be found. Here we simply make references to the equations to be used.

For simplicity, we define

$$
F_{n}=2 E_{\nu}\left(24 \pi_{B} x_{n}^{\nu}\right), \quad G_{n}=\left|\bar{e}_{2}\right|+2 \max _{3 \leq k \leq n} \sum_{j=3}^{k} P_{k-j}^{k}\left|\tilde{r}_{j}(\Delta x)\right| .
$$

Then both $F_{n}$ and $G_{n}$ are monotonically increasing with respect to $n$. Below we are going to prove the lemma using mathematical induction. Since $E_{\nu}(z)>1$ for all $z>0$, it is obvious that (4.25) holds for $n=2$. Now we assume that $n>2$ and the estimation (4.25) holds for all $\bar{e}_{2}, \ldots, \bar{e}_{n-1}$. Let

$$
\left|\bar{e}_{k(n)}\right|=\max _{2 \leq j \leq n-1}\left|\bar{e}_{j}\right|
$$

If $\left|\bar{e}_{n}\right| \leq\left|\bar{e}_{k(n)}\right|$, then the monotonicity of $F_{n}$ and $G_{n}$ shows that

$$
\left|\bar{e}_{n}\right| \leq\left|\bar{e}_{k(n)}\right| \leq F_{k(n)} G_{k(n)} \leq F_{n} G_{n} .
$$

If $\left|\bar{e}_{n}\right|>\left|\bar{e}_{k(n)}\right|$, then by the inequality (4.22)

$$
\begin{aligned}
\left|\bar{e}_{n}\right|^{2} \leq\left|\bar{e}_{n}\right| & \left(\sum_{j=3}^{n-1} P_{n-j}^{n} \sum_{k=3}^{j} 4 L \theta^{j-k}\left|\bar{e}_{k}\right|+\left|\bar{e}_{2}\right|\right. \\
& \left.+P_{0}^{n} \sum_{k=3}^{n} 4 L \theta^{n-k}\left|\bar{e}_{n}\right|+2 \sum_{j=3}^{n} P_{n-j}^{n} \cdot\left|\tilde{r}_{j}(\Delta x)\right|\right) .
\end{aligned}
$$


Using (B.1), (4.24) and $\theta<\frac{2}{3}$, we have

$$
P_{0}^{n} \sum_{k=3}^{n} 4 L \theta^{n-k}<\pi_{B} \Delta x^{\nu} \cdot 12 L \leq \frac{1}{2} .
$$

Thus according to (4.26), we can estimate $\bar{e}_{n}$ as follows:

$$
\begin{aligned}
\left|\bar{e}_{n}\right| & \leq 2\left(\sum_{j=3}^{n-1} P_{n-j}^{n} \sum_{k=3}^{j} 4 L \theta^{j-k}\left|\bar{e}_{k}\right|+\left|\bar{e}_{2}\right|+2 \sum_{j=3}^{n} P_{n-j}^{n} \cdot\left|\tilde{r}_{j}(\Delta x)\right|\right) \\
& \leq 2 \sum_{j=3}^{n-1} P_{n-j}^{n} \sum_{k=3}^{j} 4 L \theta^{j-k} F_{k} G_{k}+2 G_{n} \leq 2 \sum_{j=3}^{n-1} P_{n-j}^{n} \sum_{k=3}^{j} 4 L \theta^{j-k} F_{j} G_{n}+2 G_{n} \\
& \leq\left(24 L \sum_{j=3}^{n-1} P_{n-j}^{n} F_{j}+2\right) G_{n}=\left(48 L \sum_{j=3}^{n-1} P_{n-j}^{n} E_{\nu}\left(24 \pi_{B} L x_{j}^{\nu}\right)+2\right) G_{n} .
\end{aligned}
$$

Finally, we use (B.7) to find that

$$
\left|\bar{e}_{n}\right| \leq\left(48 \pi_{B} L \frac{E_{\nu}\left(24 \pi_{B} L x_{n}^{\nu}\right)-1}{24 \pi_{B} L}+2\right) G_{n}=2 E_{\nu}\left(24 \pi_{B} L x_{n}^{\nu}\right) G_{n}=F_{n} G_{n} .
$$

Thus the lemma is proven by the principle of mathematical induction.

Our final error estimation can be achieved by combining the above result with our estimation of the truncation error, and the conclusion is given in the following theorem:

Theorem 4.3. Let $y$ be the exact solution of (1.1) and (1.2), and $\left\{y_{k}\right\}_{k=0}^{2 N}$ be the numerical solution obtained by (2.12). Assume $y(x) \in C^{3}[0, T]$. If the step size $\Delta x$ satisfies

$$
\Delta x^{\nu} \leq \frac{1}{24 \pi_{B} L},
$$

then there exists a constant $K$ depending on $\nu, L$ and the final time $T$, such that

$$
\left|y\left(x_{k}\right)-y_{k}\right| \leq K \Delta x^{3-\nu} \quad \text { for } \quad k=1, \ldots, 2 N .
$$

Proof. Combining (B.2), (4.16) and (1.3), we have

$$
\begin{aligned}
\sum_{j=3}^{k} P_{k-j}^{k}\left|\tilde{r}_{j}(\Delta x)\right| & \leq\left(\max _{1 \leq j \leq k} \frac{1}{\omega_{1-\nu}\left(x_{j}\right)}\right) \sum_{j=1}^{k} P_{k-j}^{k} \omega_{1-\nu}\left(x_{j}\right)\left|\tilde{r}_{j}(\Delta x)\right| \\
& \leq \Gamma(1-\nu) x_{k}^{\nu} \cdot C \Delta x^{3-\nu} \sum_{j=1}^{k} P_{k-j}^{k} \omega_{1-\nu}\left(x_{j}\right) \\
& \leq\left[C \pi_{B} \Gamma(1-\nu) x_{k}^{\nu}\right] \Delta x^{3-\nu} .
\end{aligned}
$$


Substituting this estimate into (4.25) yields

$$
\begin{aligned}
\left|\bar{e}_{n}\right| & \leq 2 E_{\nu}\left(24 \pi_{B} L x_{n}^{\nu}\right)\left(\left|\bar{e}_{2}\right|+\left[2 C \pi_{B} \Gamma(1-\nu) x_{n}^{\nu}\right] \Delta x^{3-\nu}\right) \\
& \leq 2 E_{\nu}\left(24 \pi_{B} L x_{n}^{\nu}\right)\left(\left|e_{1}\right|+\left|e_{2}\right|+\left[2 C \pi_{B} \Gamma(1-\nu) x_{n}^{\nu}\right] \Delta x^{3-\nu}\right) \\
& \leq 2 E_{\nu}\left(24 \pi_{B} L x_{n}^{\nu}\right)\left(10 C_{1}+2 C \pi_{B} \Gamma(1-\nu) x_{n}^{\nu}\right) \Delta x^{3-\nu}, \text { for } n \geq 2,
\end{aligned}
$$

where we have used the estimation (4.15). Therefore, the numerical error $\left|e_{k}\right|$ can be estimated by

$$
\left|e_{k}\right|=\left|\sum_{n=0}^{k} \theta^{k-n} \bar{e}_{n}\right| \leq 3 \max _{0 \leq n \leq k} \bar{e}_{n} \leq 6 E_{\nu}\left(24 \pi_{B} L x_{k}^{\nu}\right)\left(10 C_{1}+2 C \pi_{B} \Gamma(1-\nu) x_{k}^{\nu}\right) \Delta x^{3-\nu} .
$$

The proof is completed.

\section{Numerical results}

In this section, we present numerical experiments to verify the theoretical results obtained in the previous sections.

Example 5.1. We consider the problem (1.1) with

$$
f(x, y(x))=\frac{\Gamma(4+\nu)}{6} x^{3}, \quad y(0)=0,
$$

where $f$ is independent of $y$. It can be verified that the exact solution is $y(x)=x^{3+\nu}$. The computation is carried out up to $T=1$. In our tests, we choose $\nu=0.3,0.5,0.8$, 0.99 , and for all choices of $\nu$, we choose the step size to be $\Delta x=\frac{1}{2^{l}}, l=3, \ldots, 10$. The error we will display is defined by

$$
e_{\Delta x}=\max _{k=1, \ldots, 2 N}\left|y\left(x_{k}\right)-y_{k}\right|
$$

where $2 N=\frac{T}{\Delta x}$.

By this example, we would like to check the convergence order of the numerical method with respect to the order of the fractional derivative $\nu$. The results are given in Table 1, where the convergence order is computed by $\log _{2}\left(\frac{e_{2 \Delta x}}{e_{\Delta x}}\right)$. By Theorem 4.3, we expect that this number is close to $3-\nu$. It is obvious that our numerical results are consistent with the theoretical analysis.

Example 5.2. In this example, we add the dependence on $y$ to the right-hand side $f(x, y)$. The following two functions are considered:

$$
\begin{aligned}
& f(x, y(x))=\frac{\Gamma(4+\nu)}{6} x^{3}+x^{3+\nu}-y(x), \\
& f(x, y(x))=\frac{\Gamma(4+\nu)}{6} x^{3}+x^{6+2 \nu}-y^{2}(x) .
\end{aligned}
$$


This two right-hand sides correspond to linear and nonlinear dependencies on $y$. With the initial condition $y(0)=0$, the exact solution of both is $y(x)=x^{3+\nu}$.

We take $T=1$ again and repeat the calculation in Example 5.1. The numerical error is provided in Tables 2 and 3. Due to the sufficient smoothness of the numerical solution, we again observe a good agreement with the theoretical convergence order. In particular, it is worth emphasizing that the non-linearity of $f$ seems to have no impact on the numerical order of the scheme.

Table 1: Maximum error $e_{\Delta x}$ and convergence order for Example 5.1.

\begin{tabular}{||ccccccccc||}
\hline$\Delta x$ & $\nu=0.3$ & order & $\nu=0.5$ & order & $\nu=0.8$ & order & $\nu=0.99$ & order \\
\hline$\frac{1}{8}$ & $1.6782 e-3$ & - & $5.8967 e-3$ & - & $2.3580 e-2$ & - & $4.7431 e-2$ & - \\
$\frac{1}{16}$ & $2.7683 e-4$ & 2.5998 & $1.1467 e-3$ & 2.3623 & $5.8213 e-3$ & 2.0181 & $1.3486 e-2$ & 1.8143 \\
$\frac{1}{32}$ & $4.3876 e-5$ & 2.6575 & $2.1076 e-4$ & 2.4438 & $1.3329 e-3$ & 2.1267 & $3.5413 e-3$ & 1.9291 \\
$\frac{1}{64}$ & $6.8430 e-6$ & 2.6807 & $3.7908 e-5$ & 2.4750 & $2.9674 e-4$ & 2.1673 & $9.0195 e-4$ & 1.9731 \\
$\frac{1}{128}$ & $1.0596 e-6$ & 2.6910 & $6.7551 e-6$ & 2.4884 & $6.5272 e-5$ & 2.1846 & $2.2667 e-4$ & 1.9924 \\
$\frac{1}{256}$ & $1.6356 e-7$ & 2.6957 & $1.1986 e-6$ & 2.4945 & $1.4278 e-5$ & 2.1926 & $5.6613 e-5$ & 2.0014 \\
$\frac{1}{512}$ & $2.5195 e-8$ & 2.6986 & $2.1228 e-7$ & 2.4974 & $3.1153 e-6$ & 2.1963 & $1.4096 e-5$ & 2.0057 \\
$\frac{1}{1024}$ & $3.8778 e-9$ & 2.6998 & $3.7565 e-8$ & 2.4985 & $6.7888 e-7$ & 2.1981 & $3.5049 e-6$ & 2.0078 \\
\hline
\end{tabular}

Table 2: Maximum error $e_{\Delta x}$ and convergence order for the right-hand side (5.1).

\begin{tabular}{||ccccccccc||}
\hline$\Delta x$ & $\nu=0.3$ & order & $\nu=0.5$ & order & $\nu=0.8$ & order & $\nu=0.99$ & order \\
\hline$\frac{1}{8}$ & $8.9242 e-4$ & - & $3.4577 e-3$ & - & $1.6357 e-2$ & - & $3.6070 e-2$ & - \\
$\frac{1}{16}$ & $1.4371 e-4$ & 2.6345 & $6.5136 e-4$ & 2.4083 & $3.9150 e-3$ & 2.0628 & $1.0036 e-2$ & 1.8455 \\
$\frac{1}{32}$ & $2.2556 e-5$ & 2.6715 & $1.1826 e-4$ & 2.4614 & $8.8578 e-4$ & 2.1439 & $2.6115 e-3$ & 1.9422 \\
$\frac{1}{64}$ & $3.5029 e-6$ & 2.6868 & $2.1163 e-5$ & 2.4824 & $1.9621 e-4$ & 2.1744 & $6.6251 e-4$ & 1.9788 \\
$\frac{1}{128}$ & $5.4140 e-7$ & 2.6937 & $3.7628 e-6$ & 2.4916 & $4.3066 e-5$ & 2.1878 & $1.6619 e-4$ & 1.9950 \\
$\frac{1}{256}$ & $8.3492 e-8$ & 2.6969 & $6.6703 e-7$ & 2.4959 & $9.4114 e-6$ & 2.1940 & $4.1471 e-5$ & 2.0026 \\
$\frac{1}{512}$ & $1.2854 e-8$ & 2.6993 & $1.1806 e-7$ & 2.4981 & $2.0524 e-6$ & 2.1970 & $1.0322 e-5$ & 2.0063 \\
$\frac{1}{1024}$ & $1.9781 e-9$ & 2.7000 & $2.0887 e-8$ & 2.4989 & $4.4715 e-7$ & 2.1984 & $2.5659 e-6$ & 2.0081 \\
\hline
\end{tabular}

Table 3: Maximum error $e_{\Delta x}$ and convergence order for the right-hand side (5.2).

\begin{tabular}{||ccccccccc||}
\hline$\Delta x$ & $\nu=0.3$ & order & $\nu=0.5$ & order & $\nu=0.8$ & order & $\nu=0.99$ & order \\
\hline$\frac{1}{8}$ & $9.1405 e-4$ & - & $3.2126 e-3$ & - & $1.5357 e-2$ & - & $3.4906 e-2$ & - \\
$\frac{1}{16}$ & $1.6188 e-4$ & 2.4972 & $6.4829 e-4$ & 2.3090 & $3.8037 e-3$ & 2.0134 & $1.0094 e-2$ & 1.7898 \\
$\frac{1}{32}$ & $2.6226 e-5$ & 2.6258 & $1.2091 e-4$ & 2.4226 & $8.7214 e-4$ & 2.1247 & $2.6623 e-3$ & 1.9228 \\
$\frac{1}{64}$ & $4.1349 e-6$ & 2.6651 & $2.1873 e-5$ & 2.4667 & $1.9417 e-4$ & 2.1672 & $6.7852 e-4$ & 1.9722 \\
$\frac{1}{128}$ & $6.4327 e-7$ & 2.6843 & $3.9072 e-6$ & 2.4849 & $4.2704 e-5$ & 2.1848 & $1.7050 e-4$ & 1.9925 \\
$\frac{1}{256}$ & $9.9504 e-8$ & 2.6926 & $6.9413 e-7$ & 2.4928 & $9.3407 e-6$ & 2.1927 & $4.2578 e-5$ & 2.0016 \\
$\frac{1}{512}$ & $1.5350 e-8$ & 2.6964 & $1.2299 e-7$ & 2.4965 & $2.0379 e-6$ & 2.1964 & $1.0600 e-5$ & 2.0059 \\
$\frac{1}{1024}$ & $2.3643 e-9$ & 2.6987 & $2.1774 e-8$ & 2.4978 & $4.4407 e-7$ & 2.1982 & $2.6356 e-6$ & 2.0079 \\
\hline
\end{tabular}


Example 5.3. In this example, we consider the problem with right-hand side $f(x, y)=$ $\lambda y(x)$, where $\lambda<0$ is a constant. The exact solution is $y(x)=y_{0} E_{\nu}\left(\lambda x^{\nu}\right)$, and $E_{\nu}(\cdot)$ is the Mittag-Leffler function defined in (B.8). When $\nu=1$, the fractional derivative reduces to the ordinary derivative, and the exact solution turns out to be $y(x)=y_{0} e^{\lambda x}$.

In our test, we set $\lambda=-1$ and choose the initial value $y_{0}=1$. The choices of the fractional order are now taken as $\nu=0.3,0.6,0.9,1.0$. Other settings are the same as previous two examples. When $\nu=1.0$, our numerical method reduces to the secondorder backward differentiation formula (BDF2). Results are given in Table 4, from which we can observe that when $\nu<1$, the convergence order is close to $\nu$. The reason lies in the singularity of the Mittag-Leffler function at $x=0$. When $\nu=1$, the singularity disappears, and the convergence order $3-\nu$ is restored.

Table 4: Maximum error $e_{\Delta x}$ and convergence order for Example 5.3.

\begin{tabular}{||ccccccccc||}
\hline$\Delta x$ & $\nu=0.3$ & order & $\nu=0.6$ & order & $\nu=0.9$ & order & $\nu=1.0$ & order \\
\hline$\frac{1}{8}$ & $3.2510 e-3$ & - & $8.8351 e-4$ & - & $2.1988 e-3$ & - & $3.8804 e-4$ & - \\
$\frac{1}{16}$ & $2.8864 e-3$ & 0.1716 & $6.6298 e-4$ & 0.4143 & $9.7373 e-4$ & 1.1751 & $2.9709 e-4$ & 0.3853 \\
$\frac{1}{32}$ & $2.5263 e-3$ & 0.1922 & $4.6140 e-4$ & 0.5229 & $4.5730 e-4$ & 1.0903 & $9.7657 e-5$ & 1.6051 \\
$\frac{1}{64}$ & $2.1840 e-3$ & 0.2100 & $3.1026 e-4$ & 0.5725 & $2.2952 e-4$ & 0.9945 & $2.7213 e-5$ & 1.8434 \\
$\frac{1}{128}$ & $1.8684 e-3$ & 0.2252 & $2.0569 e-4$ & 0.5930 & $1.2005 e-4$ & 0.9350 & $7.1461 e-6$ & 1.9290 \\
$\frac{1}{256}$ & $1.5842 e-3$ & 0.2380 & $1.3568 e-4$ & 0.6003 & $6.3888 e-5$ & 0.9100 & $1.8289 e-6$ & 1.9661 \\
$\frac{1}{512}$ & $1.3332 e-3$ & 0.2488 & $8.9370 e-5$ & 0.6023 & $3.4223 e-5$ & 0.9006 & $4.6252 e-7$ & 1.9834 \\
$\frac{1}{1024}$ & $1.1150 e-3$ & 0.2578 & $5.8861 e-5$ & 0.6025 & $1.8362 e-5$ & 0.8982 & $1.1628 e-7$ & 1.9918 \\
\hline
\end{tabular}

Table 5: Maximum error and convergence order of the corrected method for Example 5.3.

\begin{tabular}{||ccccccc||}
\hline$\Delta x$ & $\nu=0.3$ & order & $\nu=0.6$ & order & $\nu=0.9$ & order \\
\hline$\frac{1}{8}$ & $2.4932 e-6$ & - & $4.2141 e-5$ & - & $1.2940 e-4$ & - \\
$\frac{1}{16}$ & $8.5679 e-7$ & 1.5409 & $1.7729 e-5$ & 1.2491 & $7.0189 e-5$ & $8.8254 e-01$ \\
$\frac{1}{32}$ & $2.8365 e-7$ & 1.5947 & $5.0652 e-6$ & 1.8074 & $2.3691 e-5$ & 1.5668 \\
$\frac{1}{64}$ & $9.0097 e-8$ & 1.6546 & $1.2249 e-6$ & 2.0479 & $6.6215 e-6$ & 1.8391 \\
$\frac{1}{128}$ & $2.7462 e-8$ & 1.7140 & $2.7037 e-7$ & 2.1796 & $1.6940 e-6$ & 1.9667 \\
$\frac{1}{256}$ & $8.0536 e-9$ & 1.7697 & $5.6509 e-8$ & 2.2583 & $4.1466 e-7$ & 2.0304 \\
$\frac{1}{512}$ & $2.2805 e-9$ & 1.8202 & $1.1354 e-8$ & 2.3152 & $9.9291 e-8$ & 2.0622 \\
$\frac{1}{1024}$ & $6.2613 e-10$ & 1.8648 & $2.5311 e-9$ & 2.1654 & $2.3508 e-8$ & 2.0785 \\
\hline
\end{tabular}

The convergence order can be improved by Lubich's method [29] to include singular terms in the ansatz of the solution. This is achieved by choosing a finite sequence of positive real numbers $\sigma_{1}<\ldots<\sigma_{m+1}$, and assume that

$$
y(x)-y(0)=\sum_{j=1}^{m} c_{j} x^{\sigma_{j}}+x^{\sigma_{m+1}} \tilde{y}(x),
$$


where $\tilde{y}(x)$ is a bounded function, and we assume that the term $x^{\sigma_{m+1}} \tilde{y}(x)$ is sufficiently smooth to retain our convergence order. The sum of $c_{j} x^{\sigma_{j}}$ captures the less smooth part, for which the discretization of the fractional derivative needs to be altered to get better accuracy. Here we omit the detailed derivation, and refer the readers to $[29,41,42]$ for more discussions on the correction method. The final numerical scheme discretizes the fractional derivative by

$$
{ }_{0} D_{\Delta x}^{\nu, m} y_{n}={ }_{0} D_{\Delta x}^{\nu} y_{n}+\Delta x^{-\nu} \sum_{j=1}^{m} W_{n, j}\left(y_{j}-y_{0}\right),
$$

where $W_{n, j}$ are the starting weights that are chosen such that

$$
\begin{aligned}
& { }_{0} D_{\Delta x}^{\nu} q_{k}\left(x_{n}\right)+\Delta x^{-\nu} \sum_{j=1}^{m} W_{n, j} q_{k}\left(x_{j}\right) \\
= & \frac{\Gamma\left(1+\sigma_{k}\right)}{\Gamma\left(1-\nu+\sigma_{k}\right)} x_{n}^{\sigma_{k}-\nu} \text { for all } k=1, \ldots, m,
\end{aligned}
$$

where $q_{k}(x)=x^{\sigma_{k}}$.

In this example, we choose $\sigma_{k}=k \nu$. Then $W_{n, j}, 1 \leq j \leq m$ can be solved from (5.5), and the values of $W_{n, j}$ are independent of $\Delta x$. Since the series expansion of the exact solution includes terms such as $x^{\nu}$ and $x^{2 \nu}$, Lubich's correction method is suitable for such a problem. The results of the corrected method are given in Table 5, which shows remarkable improvement compared with Table 4.

\section{Conclusion}

An efficient high-order approximate numerical scheme for fractional ordinary differential equations with the Caputo derivative has been introduced in this paper. The scheme is unconditionally stable and has uniform accuracy for all time steps. The proof of stability shows the technical details on how to deal with the special initial steps. The sharp numerical order $3-\nu$ is proven for sufficiently smooth solutions and general nonlinear equations, and this order is verified by our numerical experiments. For solutions with initial singularity, our scheme can couple with Lubich's method seamlessly to improve the numerical order. Future works include the construction and analysis of the scheme for graded meshes to resolve the initial layer.

\section{Acknowledgements}

This research was supported by National Natural Science Foundation of China (Nos. 11901135, 11961009), Foundation of Guizhou Science and Technology Department (Nos. [2020]1Y015, [2017]1086). The first author would like to acknowledge the financial support by the China Scholarship Council (201708525037). The second author was supported by the Academic Research Fund of the Ministry of Education of Singapore under grant No. R-146-000-305-114. 


\section{Appendix A: Proof of some inequalities}

In this appendix, we provide the proofs of two lemmas used in the stability analysis, which include a number of technical inequalities.

Lemma A.1. For any $k \geq 2$, it holds that
(1) $\left(1-\frac{1}{k}\right)^{1-\nu}+\left(1+\frac{1}{k}\right)^{1-\nu} \geq 2-\frac{1-\nu}{2 k^{2}}\left[2^{\nu}-\left(\frac{2}{3}\right)^{\nu}\right]$,
(2) $\left(1-\frac{1}{k}\right)^{2-\nu}-\left(1+\frac{1}{k}\right)^{2-\nu} \geq-2(2-\nu) \frac{1}{k}+\frac{1}{3 k^{3}}(2-\nu)(1-\nu) \nu$,
(3) $\frac{2-\nu}{2} \frac{1}{2 k}\left[\left(1-\frac{2}{2 k}\right)^{1-\nu}+3-4\left(1+\frac{1}{2 k}\right)^{1-\nu}\right]+\left(1-\frac{2}{2 k}\right)^{2-\nu}$ $-3+2\left(1+\frac{1}{2 k}\right)^{2-\nu} \geq 0$
(4) $-\nu^{2}-12+3\left(\frac{2}{3}\right)^{\nu}\left(\nu^{2}+2 \nu+4\right)>0$,
(5) $6-\nu-\left(2+\frac{\nu}{2}\right) 2^{\nu} 3^{1-\nu}<0$
(6) $-2 \nu^{3}+12 \nu^{2}-56 \nu-48+3\left(\frac{2}{3}\right)^{\nu}\left(3 \nu^{3}+4 \nu^{2}+20 \nu+16\right)<0$,
(7) $2^{1-\nu}\left[4-\nu-(2+\nu) 2^{1-\nu}\right]<\frac{1}{27}(2 \nu-3)(2-\nu)(1-\nu) \nu$,
(8) $12-\nu^{2}-\left(12+8 \nu+\nu^{2}\right) 2^{-\nu}>\frac{1}{16}(2+\nu)(2-\nu)(1-\nu) \nu$.

Proof. (1) This inequality is equivalent to

$$
f_{1}(t):=(1-t)^{1-\nu}+(1+t)^{1-\nu}-2\left(1-A t^{2}\right) \geq 0
$$

for $t=\frac{1}{k}$ and $A=\frac{1}{4}(1-\nu)\left[2^{\nu}-\left(\frac{2}{3}\right)^{\nu}\right]$. Since $k \geq 2$, the range of $t$ is $\left(0, \frac{1}{2}\right]$. To show (A.1), we take the derivative of $f_{1}(t)$ to get

$$
f_{1}^{\prime}(t)=-(1-\nu)\left[(1-t)^{-\nu}-(1+t)^{-\nu}\right]+4 A t=-4 t\left[f_{2}(t)-A\right],
$$

where

$$
\begin{aligned}
f_{2}(t) & =\frac{1-\nu}{4} \frac{(1-t)^{-\nu}-(1+t)^{-\nu}}{t} \\
& =\frac{1-\nu}{4} \sum_{j=0}^{+\infty} \frac{2}{(2 j+1) !} \nu(1+\nu) \ldots(2 j+\nu) t^{2 j} .
\end{aligned}
$$

The series expansion of $f_{2}$ clearly shows that $f_{2}$ is an increasing function, which yields $f_{2}(t) \leq f_{2}\left(\frac{1}{2}\right)=A$. Thus by (A.2), we have $f_{1}^{\prime}(t) \geq 0$, indicating that

$$
f_{1}(t) \geq f_{1}(0)=0 \text {. }
$$


(2) This inequality can be similarly proven by defining

$$
f_{3}(t)=(1-t)^{2-\nu}-(1+t)^{2-\nu}+2(2-\nu) t-\frac{1}{3}(2-\nu)(1-\nu) \nu t^{3},
$$

whose series expansion is

$$
f_{3}(t)=\sum_{j=2}^{+\infty} \frac{2}{(2 j+1) !}(-2+\nu)(-1+\nu) \nu(1+\nu) \ldots(2 j-2+\nu) t^{2 j+1} .
$$

Since all the terms in the sum are monotonically increasing, we have $f_{3}(t) \geq f_{3}(0)=0$. The proof is completed by setting $t=\frac{1}{k}$.

(3) Let

$$
f_{1}(k, \nu)=\left(1-\frac{2}{2 k}\right)^{2-\nu}+2\left(1+\frac{1}{2 k}\right)^{2-\nu}, \quad f_{2}(k, \nu)=\left(1-\frac{2}{2 k}\right)^{2-\nu} .
$$

Then the desired inequality is equivalent to

$$
f(k, \nu):=\left(1-\frac{2-\nu}{2 k+1}\right) f_{1}(k, \nu)+\left[\frac{2-\nu}{4(k-1)}+\frac{2-\nu}{2 k+1}\right] f_{2}(k, \nu)+\frac{3(2-\nu)}{4 k}-3 \geq 0 .
$$

Since $k \geq 2$, we can apply binomial expansion to $f_{1}$ to obtain

$$
f_{1}(k, \nu)=\sum_{j=0}^{+\infty}\left(\begin{array}{c}
2-\nu \\
j
\end{array}\right)\left[(-2)^{j}+2\right]\left(\frac{1}{2 k}\right)^{j} .
$$

It can be observed that when $j \geq 2$, the summand in the above sum is positive. Therefore

$$
\begin{aligned}
f_{1}(k, \nu) & \geq \sum_{j=0}^{3}\left(\begin{array}{c}
2-\nu \\
j
\end{array}\right)\left[(-2)^{j}+2\right]\left(\frac{1}{2 k}\right)^{j} \\
& =3+\frac{3(2-\nu)(1-\nu)}{4 k^{2}}+\frac{(2-\nu)(1-\nu) \nu}{8 k^{3}}
\end{aligned}
$$

By similar means, we get

$$
f_{2}(k, \nu) \geq 1-\frac{2-\nu}{k}+\frac{(2-\nu)(1-\nu)}{2 k^{2}}+\frac{(2-\nu)(1-\nu) \nu}{6 k^{3}} .
$$

Plugging (A.4) and (A.5) into the expression of the $f(k, \nu)$, we get

$$
\begin{aligned}
f(k, \nu) & \geq \frac{(2-\nu)(1-\nu) \nu}{8 k^{3}(k-1)(2 k+1)}[-3 k+(k-1)(2 k-1+\nu)+(2 k-1)(2-\nu)] \\
& \geq \frac{(2-\nu)(1-\nu) \nu}{8 k^{3}(k-1)(2 k+1)}[-3 k+(k-1)(3+\nu)+3(2-\nu)] \\
& =\frac{(2-\nu)(1-\nu) \nu}{8 k^{3}(k-1)(2 k+1)}[(k-1) \nu+3(1-\nu)] \geq 0
\end{aligned}
$$


which completes the proof.

(4) Let

$$
\tilde{h}(\nu)=-\nu^{2}-12+3\left(\frac{2}{3}\right)^{\nu}\left(\nu^{2}+2 \nu+4\right) .
$$

Then its first-order derivative is

$$
\tilde{h}^{\prime}(\nu)=-2 \nu+3\left(\frac{2}{3}\right)^{\nu}\left[2 \nu+2+\left(\nu^{2}+2 \nu+4\right) \log \frac{2}{3}\right] .
$$

When $\nu \in(0,1)$, by the property of quadratic functions, one can show that

$$
2 \nu+2+\left(\nu^{2}+2 \nu+4\right) \log \left(\frac{2}{3}\right)>0
$$

Therefore

$$
\begin{aligned}
\tilde{h}^{\prime}(\nu) & \geq-2 \nu+2\left[2 \nu+2+\left(\nu^{2}+2 \nu+4\right) \log \frac{2}{3}\right] \\
& =2\left[\nu+2+\left(\nu^{2}+2 \nu+4\right) \log \frac{2}{3}\right] .
\end{aligned}
$$

Again it can be shown by the property of quadratic functions that $\tilde{h}^{\prime}(\nu)>0$ for all $\nu \in(0,1)$. Thus

$$
\tilde{h}(\nu)>\tilde{h}(0)=0 .
$$

(5) Let

$$
g(\nu)=6-\nu-\left(2+\frac{\nu}{2}\right) 2^{\nu} 3^{1-\nu} .
$$

We want to prove $g(\nu)<0$. The first-order and second-order derivatives of $g$ are

$$
\begin{aligned}
& g^{\prime}(\nu)=-1-\left(\frac{2}{3}\right)^{\nu-1}-\left(6+\frac{3}{2} \nu\right)\left(\frac{2}{3}\right)^{\nu} \log \frac{2}{3} \\
& g^{\prime \prime}(\nu)=-\left(\frac{2}{3}\right)^{\nu-1}\left(2+\log \frac{16}{81}+\nu \log \frac{2}{3}\right) \log \frac{2}{3}
\end{aligned}
$$

It is clear that $g^{\prime \prime}(\nu)$ changes from positive to negative as $\nu$ varies from 0 to 1 . Therefore $g^{\prime}(\nu)$ first increases and then decreases. By straightforward calculation, we see that $g^{\prime}(0)<0$ and $g^{\prime}(1)>0$, meaning that $g(\nu)$ first decreases and then increases. Therefore

$$
g(\nu)<\max (g(0), g(1))=0 .
$$


(6) Let $\tilde{g}(\nu)$ be the left-hand side. Then the fourth-order derivative of $\tilde{g}(\nu)$ is

$$
\begin{gathered}
\tilde{g}^{(4)}(\nu)=3\left(\frac{2}{3}\right)^{\nu} \log \frac{2}{3}\left[72+6(18 \nu+8) \log \frac{2}{3}+4\left(9 \nu^{2}+8 \nu+20\right)\left(\log \frac{2}{3}\right)^{2}\right. \\
\left.+\left(3 \nu^{3}+4 \nu^{2}+20 \nu+16\right)\left(\log \frac{2}{3}\right)^{3}\right] \\
<3\left(\frac{2}{3}\right)^{\nu} \log \frac{2}{3}\left[72+156 \log \frac{2}{3}+80\left(\log \frac{2}{3}\right)^{2}+43\left(\log \frac{2}{3}\right)^{3}\right]<0
\end{gathered}
$$

Therefore $\tilde{g}^{\prime \prime \prime}(\nu)$ is monotonically decreasing for $\nu \in(0,1)$. Straightforward calculation yields $\tilde{g}^{\prime \prime \prime}(0)>0$ and $\tilde{g}^{\prime \prime \prime}(1)<0$, which indicates that $\tilde{g}^{\prime \prime}(\nu)$ first increases and then decreases. Since $\tilde{g}^{\prime \prime}(0)>0$ and $\tilde{g}^{\prime \prime}(1)>0$, we know that $\tilde{g}^{\prime}(\nu)$ increases monotonically. Finally, using $\tilde{g}^{\prime}(0)<0$ and $\tilde{g}^{\prime}(1)>0$, one sees that $\tilde{g}(\nu)$ first decreases and then increases, which implies

$$
\tilde{g}(\nu)<\max (\tilde{g}(0), \tilde{g}(1))=0 .
$$

This completes the proof.

(7) This inequality can be proven using the same method as (6).

(8) Define

$$
g(\nu)=\frac{1}{16}(2+\nu)(2-\nu)(1-\nu) \nu-\left[12-\nu^{2}-\left(12+8 \nu+\nu^{2}\right) 2^{-\nu}\right] .
$$

The third-order derivative of $g$ satisfies

$$
\begin{aligned}
g^{(3)}(\nu)= & 2^{-3-\nu}\left[2^{\nu}(12 \nu-3)-48 \log 2+(192+48 \nu)(\log 2)^{2}\right. \\
& \left.-\left(8 \nu^{2}+64 \nu+96\right)(\log 2)^{3}\right] \\
> & 2^{-3-\nu}\left[-3-48 \log 2+192(\log 2)^{2}-168(\log 2)^{3}\right]>0
\end{aligned}
$$

which means $g^{\prime \prime}(\nu)$ is monotonically increasing. Using $g^{\prime \prime}(1)<0$, we know that $g^{\prime}(\nu)$ is an decreasing function. Finally, using $g^{\prime}(0)<0$, we know that $g^{\prime}(\nu)$ is negative for all $\nu \in(0,1)$. Thus $g(\nu)<g(0)=0$.

Lemma A.2. Suppose $0<b<2 m$. Let

$$
f(\nu)=(2-\nu)\left[a_{1}(2 m)^{1-\nu}+a_{2}(2 m+b)^{1-\nu}\right]+a_{3}\left[(2 m)^{2-\nu}-(2 m+b)^{2-\nu}\right] .
$$

Then we have

1. If $\frac{a_{3} b}{a_{2}} \leq \frac{3}{2}$ and $a_{2}<0$, then

$$
\begin{aligned}
f(\nu) & <(2-\nu)(2 m)^{1-\nu}\left[a_{1}+a_{2}-a_{3} b+\sum_{k=1}^{2}\left(\begin{array}{c}
1-\nu \\
k
\end{array}\right)\left(a_{2}-\frac{a_{3} b}{k+1}\right) \frac{b^{k}}{(2 m)^{k}}\right] \\
& <(2-\nu)(2 m)^{1-\nu}\left(a_{1}+a_{2}-a_{3} b\right) .
\end{aligned}
$$


2. If $a_{3} b=2 a_{2}>0$, then

$$
f(\nu)<(2-\nu)(2 m)^{1-\nu}\left[a_{1}+a_{2}-a_{3} b-a_{2}\left(\frac{b}{2 m}\right)^{2} \frac{(1-\nu) \nu}{6}\left(1-\frac{\nu+1}{2} \frac{b}{2 m}\right)\right] .
$$

Proof. Since $b<2 m$, we can apply binomial expansion to get

$$
\begin{aligned}
f(\nu) & =(2-\nu)(2 m)^{1-\nu}\left[a_{1}+a_{2}\left(1+\frac{b}{2 m}\right)^{1-\nu}\right]+a_{3}(2 m)^{2-\nu}\left[1-\left(1+\frac{b}{2 m}\right)^{2-\nu}\right] \\
& =(2-\nu)(2 m)^{1-\nu}\left[a_{1}+a_{2}-a_{3} b+\sum_{k=1}^{+\infty}\left(\begin{array}{c}
1-\nu \\
k
\end{array}\right)\left(a_{2}-\frac{a_{3} b}{k+1}\right) \frac{b^{k}}{(2 m)^{k}}\right] .
\end{aligned}
$$

When $0<b<2 m$ and $\frac{a_{3} b}{a_{2}} \leq 2$, then the above series is an alternating series. Denote the above series by $\sum_{k=1}^{+\infty} S_{k}$. Then by $b<2 m$, we see that

$$
\left|S_{k+1}\right| \leq \frac{(k+\nu-1)\left(k+2-a_{3} b / a_{2}\right)}{(k+2)\left(k+1-a_{3} b / a_{2}\right)}\left|S_{k}\right|
$$

We want to show that the factor in front of $\left|S_{k}\right|$ is less than one, meaning that $\left\{\left|S_{k}\right|\right\}$ decreases monotonically. To show this, we take the difference between the numerator and the denominator:

$$
\begin{aligned}
& (k+\nu-1)\left(k+2-\frac{a_{3} b}{a_{2}}\right)-(k+2)\left(k+1-\frac{a_{3} b}{a_{2}}\right) \\
= & (2+k)(\nu-2)+\frac{a_{3} b}{a_{2}}(3-\nu) .
\end{aligned}
$$

Now we consider the two cases separately:

Case 1: If $\frac{a_{3} b}{a_{2}} \leq \frac{3}{2}$ and $a_{2}<0$, then

$$
(2+k)(\nu-2)+\frac{a_{3} b}{a_{2}}(3-\nu) \leq 3(\nu-2)+\frac{3}{2}(3-\nu)=\frac{3}{2}(\nu-1)<0 .
$$

Therefore $\left|S_{k+1}\right| \leq\left|S_{k}\right|$, indicating that the sign of the alternating series is determined by the sign of the first term. Using

$$
S_{1}=(1-\nu)\left(a_{2}-\frac{a_{3} b}{2}\right) \frac{b}{2 m}=(1-\nu) a_{2}\left(1-\frac{a_{3} b}{2 a_{2}}\right) \frac{b}{2 m}<0,
$$

we conclude that the series in (A.8) is negative. Therefore

$$
f(\nu)<(2-\nu)(2 m)^{1-\nu}\left(a_{1}+a_{2}-a_{3} b+S_{1}+S_{2}\right) .
$$


Case 2: If $a_{3} b=2 a_{2}>0$, we have $S_{1}=0$. We only need to study the sign of (A.9) when $k \geq 2$ :

$$
(2+k)(\nu-2)+\frac{a_{3} b}{a_{2}}(3-\nu) \leq 4(\nu-2)+2(3-\nu)=2(\nu-1)<0 .
$$

Therefore we also have $\left|S_{k+1}\right| \leq\left|S_{k}\right|$. Now the first term in the series is

$$
S_{2}=-\left(\frac{b}{2 m}\right)^{2} \frac{(1-\nu) \nu}{3 !} a_{2}<0 .
$$

Thus the whole series is also negative. In this case, we have

$$
f(\nu)<(2-\nu)(2 m)^{1-\nu}\left(a_{1}+a_{2}-a_{3} b+S_{2}+S_{3}\right) .
$$

The Eq. (A.7) can be obtained by inserting the expressions of $S_{2}$ and $S_{3}$.

\section{Appendix B: Some results in the proof of Lemma 4.5}

Now we provide the proof of some results used in the proof of Lemma 4.5. The proof is generally in accordance with the corresponding results in [25]. The difference is that according to our definition of $P_{j}^{n}$, the Eqs. (4.20) and (4.21) are not equalities. Consequently, the results in [25] cannot be directly applied to our case. Below we divide the proof into three lemmas.

Lemma B.1. The discrete kernels $P_{j}^{n}$ defined in (4.18) satisfy

$$
\begin{array}{ll}
0 \leq P_{n-j}^{n} \leq \pi_{B} \Gamma(2-\nu) \Delta x^{\nu}, & 3 \leq j \leq n \leq 2 N, \\
\sum_{j=3}^{n} P_{n-j}^{n} \omega_{1-\nu}\left(x_{j}\right) \leq \pi_{B}, & 3 \leq n \leq 2 N .
\end{array}
$$

Proof. According [25, Lemma 2.1], we can directly obtain (B.1). We mainly focus on (B.2). Taking $n=j$ and $k=j-1$ in (4.6), we have

$$
\begin{gathered}
\bar{B}_{j-1}^{j} \geq \frac{1}{\pi_{B} \Delta x \Gamma(2-\nu)}\left[j^{1-\nu}-(j-1)^{1-\nu}\right]=\frac{1}{\pi_{B} \Delta x \Gamma(1-\nu)} \frac{j^{1-\nu}-(j-1)^{1-\nu}}{1-\nu} \\
\geq \frac{1}{\pi_{B} \Delta x^{\nu} \Gamma(1-\nu)} j^{-\nu}=\frac{1}{\pi_{B}} \frac{x_{j}^{-\nu}}{\Gamma(1-\nu)}=\frac{1}{\pi_{B}} \omega_{1-\nu}\left(x_{j}\right),
\end{gathered}
$$

which indicates $\omega_{1-\nu}\left(x_{j}\right) \leq \pi_{B} \bar{B}_{j-1}^{j}$. By (4.17) and Lemma 4.1, we obtain

$$
\sum_{j=3}^{n} P_{n-j}^{n} \omega_{1-\nu}\left(x_{j}\right) \leq \pi_{B} \sum_{j=3}^{n} P_{n-j}^{n} \bar{B}_{j-1}^{j} \leq \pi_{B} \sum_{j=3}^{n} P_{n-j}^{n} \bar{B}_{j-3}^{j}=\pi_{B}
$$

as completes the proof. 
Lemma B.2. Let $v:[0, T] \rightarrow R$ be a continuous and piecewise $C^{1}$ function whose derivative $v^{\prime}(x)$ is nonnegative for all $x \in[0, T]$. Then

(I) If $v^{\prime}$ is monotonically decreasing, we have

$$
\sum_{j=3}^{n} P_{n-j}^{n}\left({ }_{0} D_{x}^{\nu} v\right)\left(x_{j}\right) \leq \pi_{B} \int_{0}^{x_{n}} v^{\prime}(s) d s=\pi_{B}\left[v\left(x_{n}\right)-v(0)\right], \quad 3 \leq n \leq 2 N .
$$

(II) If $v^{\prime}$ is monotonic, then

$$
\sum_{j=3}^{n-1} P_{n-j}^{n}\left({ }_{0} D_{x}^{\nu} v\left(x_{j}\right)\right) \leq \pi_{B} \int_{0}^{x_{n}} v^{\prime}(s) d s=\pi_{B}\left[v\left(x_{n}\right)-v(0)\right], \quad 3 \leq n \leq 2 N .
$$

Proof. (I) The proof requires the Chebyshev's sorting inequality [9, P.168, item 236]: if $f$ is monotone increasing and $g$ is monotone decreasing on the interval $[a, b]$, and both functions are integrable, we have

$$
(b-a) \int_{a}^{b} f(s) g(s) d s \leq \int_{a}^{b} f(s) d s \int_{a}^{b} g(s) d s .
$$

In this inequality, we set $[a, b]=\left[x_{k-1}, x_{k}\right], f(s)=w_{1-\alpha}\left(x_{j}-s\right)$ and $g(s)=v^{\prime}(s) \geq 0$. Using Lemma 4.1, we see that when $j \geq 3$,

$$
\begin{aligned}
\left({ }_{0} D_{x}^{\nu} v\right)\left(x_{j}\right) & =\int_{0}^{x_{j}} w_{1-\alpha}\left(x_{j}-s\right) v^{\prime}(s) d s=\sum_{k=1}^{j} \int_{x_{k-1}}^{x_{k}} w_{1-\alpha}\left(x_{j}-s\right) v^{\prime}(s) d s \\
& \leq \sum_{k=1}^{j} \frac{1}{\Delta x} \int_{x_{k-1}}^{x_{k}} w_{1-\alpha}\left(x_{j}-s\right) d s \int_{x_{k-1}}^{x_{k}} v^{\prime}(s) d s \\
& \leq \sum_{k=1}^{j} \pi_{B} \bar{B}_{j-k}^{j} \int_{x_{k-1}}^{x_{k}} v^{\prime}(s) d s=\pi_{B} \sum_{k=1}^{j} \bar{B}_{j-k}^{j} \int_{x_{k-1}}^{x_{k}} v^{\prime}(s) d s .
\end{aligned}
$$

Thus, from the (4.17), (4.20) and (4.21), we conclude that

$$
\begin{aligned}
& \sum_{j=3}^{n} P_{n-j}^{n}\left({ }_{0} D_{x}^{\nu} v\right)\left(x_{j}\right) \leq \sum_{j=3}^{n} P_{n-j}^{n} \pi_{B} \sum_{k=1}^{j} \bar{B}_{j-k}^{j} \int_{x_{k-1}}^{x_{k}} v^{\prime}(s) d s \\
= & \pi_{B} \sum_{k=1}^{n} \int_{x_{k-1}}^{x_{k}} v^{\prime}(s) d s \sum_{j=k}^{n} P_{n-j}^{n} \bar{B}_{j-k}^{j} \leq \pi_{B} \int_{0}^{x_{n}} v^{\prime}(s) d s .
\end{aligned}
$$

(II) Since $v^{\prime}(x) \geq 0$, we have

$$
\left({ }_{0} D_{x}^{\nu} v\right)\left(x_{j}\right)=\sum_{k=1}^{j} \int_{x_{k-1}}^{x_{k}} w_{1-\alpha}\left(x_{j}-s\right) v^{\prime}(s) d s \geq 0 .
$$


Therefore if $v^{\prime}$ is monotonically decreasing, then (B.5) is a simple corollary of (B.4). If $v^{\prime}$ is increasing, we can use Lemma 4.1 and (4.19) to obtain

$$
\begin{aligned}
& \sum_{j=3}^{n-1} P_{n-j}^{n}\left({ }_{0} D_{x}^{\nu} v\right)\left(x_{j}\right)=\sum_{j=3}^{n-1} P_{n-j}^{n} \sum_{k=1}^{j} \int_{x_{k-1}}^{x_{k}} w_{1-\alpha}\left(x_{j}-s\right) v^{\prime}(s) d s \\
\leq & \sum_{j=3}^{n-1} P_{n-j}^{n} \sum_{k=1}^{j} v^{\prime}\left(x_{k}\right) \int_{x_{k-1}}^{x_{k}} w_{1-\alpha}\left(x_{j}-s\right) d s \leq \pi_{B} \Delta x \sum_{j=3}^{n-1} P_{n-j}^{n} \sum_{k=1}^{j} v^{\prime}\left(x_{k}\right) \bar{B}_{j-k}^{j} \\
= & \pi_{B} \Delta x \sum_{j=1}^{n-1} P_{n-j}^{n} \sum_{k=1}^{j} v^{\prime}\left(x_{k}\right) \bar{B}_{j-k}^{j}=\pi_{B} \Delta x \sum_{k=1}^{n-1} v^{\prime}\left(x_{k}\right) \sum_{j=k}^{n-1} P_{n-j}^{n} \bar{B}_{j-k}^{j} \\
\leq & \pi_{B} \Delta x \sum_{k=1}^{n-1} v^{\prime}\left(x_{k}\right) \leq \pi_{B} \sum_{k=1}^{n-1} \int_{x_{k}}^{x_{k+1}} v^{\prime}(s) d s \leq \pi_{B} \int_{0}^{x_{n}} v^{\prime}(s) d s .
\end{aligned}
$$

This proves (B.5).

Lemma B.3. For the discrete kernels $P_{j}^{n}$ defined in (4.18), it holds for any $\mu>0$ that

$$
\sum_{j=3}^{n-1} P_{n-j}^{n} E_{\nu}\left(\mu x_{j}^{\nu}\right) \leq \frac{\pi_{B}}{\mu}\left[E_{\nu}\left(\mu x_{n}^{\nu}\right)-1\right], \quad 3 \leq n \leq 2 N
$$

where $E_{\nu}(\cdot)$ is the Mittag-Leffler function defined by

$$
E_{\nu}(z):=\sum_{k=0}^{+\infty} \frac{z^{k}}{\Gamma(1+k \nu)}
$$

Proof. Define

$$
v_{k}(x)=\frac{x^{k \nu}}{\Gamma(1+k \nu)}
$$

Then

$$
E_{\nu}\left(\mu x^{\nu}\right)=\sum_{k=0}^{+\infty} \frac{\mu^{k} x^{k \nu}}{\Gamma(1+k \nu)}=\sum_{k=0}^{+\infty} \mu^{k} v_{k}(x) .
$$

The function $v_{k}(x)$ satisfies

$$
\begin{aligned}
& v_{0}(x)=1, \quad v_{k}^{\prime}(x)=\frac{x^{k \nu-1}}{\Gamma(k \nu)}=\omega_{k \nu}(x), \quad v_{k}^{\prime \prime}(x)=\frac{(k \nu-1) x^{k \nu-2}}{\Gamma(k \nu)}, \\
& \left({ }_{0} D_{x}^{\nu} v_{k}\right)(x)=\int_{0}^{x} w_{1-\alpha}\left(x_{j}-s\right) \omega_{k \nu}(s) d s=\omega_{1+(k-1) \nu}(x)=v_{k-1}(x), \quad \forall k \geq 1 .
\end{aligned}
$$

Therefore for all $x>0, v_{k}^{\prime \prime}(x) \leq 0$ if $k \nu-1 \leq 0$ and $v_{k}^{\prime \prime}(x)>0$ if $k \nu-1>0$. Thus, $v_{k}^{\prime}(x)$ is non-negative and monotonic, so we can apply (B.5) to get

$$
\sum_{j=3}^{n-1} P_{n-j}^{n}\left({ }_{0} D_{x}^{\nu} v_{k}\right)\left(x_{j}\right) \leq \pi_{B}\left[v_{k}\left(x_{n}\right)-v_{k}(0)\right]=\pi_{B} v_{k}\left(x_{n}\right), \quad \forall k \geq 1 .
$$


The Eqs. (B.11) and (B.12) yield

$$
\sum_{j=3}^{n-1} P_{n-j}^{n} \sum_{k=1}^{m} \mu^{k} v_{k-1}\left(x_{j}\right) \leq \pi_{B} \sum_{k=1}^{m} \mu^{k} v_{k}\left(x_{n}\right) .
$$

Now we take the limit as $m \rightarrow+\infty$. The right-hand side of the above inequality approaches to $\pi_{B}\left(E_{\nu}\left(\mu x_{n}^{\nu}\right)-1\right)$, and the limit of the left-hand side is

$$
\sum_{j=3}^{n-1} P_{n-j}^{n} \sum_{k=1}^{+\infty} \mu^{k} v_{k-1}\left(x_{j}\right)=\sum_{j=3}^{n-1} P_{n-j}^{n} \mu \sum_{k=0}^{+\infty} \mu^{k} v_{k}\left(x_{j}\right)=\sum_{j=3}^{n-1} P_{n-j}^{n} \mu E_{\nu}\left(\mu x_{j}^{\nu}\right) .
$$

This completes the proof.

\section{References}

[1] A. A. Alikhanov, A new difference scheme for the time fractional diffusion equation, J. Comput. Phys., 280 (2015), pp. 424-438.

[2] D. BAFFET AND J. S. HESTHAVEN, High-order accurate local schemes for fractional differential equations, J. Sci. Comput., 70 (2017), pp. 355-385.

[3] J. CAO, C. LI, AND Y. Chen, High-order approximation to Caputo derivatives and Caputotype advection-diffusion equations (ii), Frac. Cal. Appl. Anal., 18 (2015), pp. 735-761.

[4] J. CAO AND C. XU, A high order schema for the numerical solution of the fractional ordinary differential equations, J. Comput. Phys., 238 (2013), pp. 154-168.

[5] M. CUI, Finite difference schemes for the variable coefficients single and multi-term timefractional diffusion equations with non-smooth solutions on graded and uniform meshes, Numer. Math. Theor. Meth. Appl., 12 (2019), pp. 845-866.

[6] K. Diethelm AND N. J. Ford, Analysis of fractional differential equations, J. Math. Anal. Appl., 265 (2002), pp. 229-248.

[7] K. DieThelM, J. M. FORD, N. J. FoRD, AND M. WEILBEER, Pitfalls in fast numerical solvers for fractional differential equations, J. Comput. Appl. Math., 186 (2006), pp. 482-503.

[8] R. DU, Y. YAN, AND Z. LIANG, A high-order scheme to approximate the Caputo fractional derivative and its application to solve the fractional diffusion wave equation, J. Comput. Phys., 376 (2019), pp. 1312-1330.

[9] G. H. HARDY, J. E. LitTleWood, AND G. PÓLYA, Inequalities, Cambridge University Press, 1934.

[10] G. GaO AND Z. Sun, A compact finite difference scheme for the fractional sub-diffusion equations, J. Comput. Phys., 230 (2011), pp. 586-595.

[11] G. GAO, Z. Sun, AND H. ZhANG, A new fractional numerical differentiation formula to approximate the Caputo fractional derivative and its applications, J. Comput. Phys., 259 (2014), pp. 33-50.

[12] R. Garrappa, E. Messina, AND A. VeCCHio, Effect of perturbation in the numerical solution of fractional differential equations., Discrete Cont. Dyn. Syst. Ser. B, 23 (2018).

[13] B. GuO, X. Pu, AND F. HuAng, Fractional Partial Differential Equations and Their Numerical Solutions, World Scientific, 2015.

[14] J. HUANG, Y. TANG, AND L. VÁZQUEZ, Convergence analysis of a block-by-block method for fractional differential equations, Numer. Math. Theor. Meth. Appl., 5 (2012), pp. 229-241. 
[15] S. JiANG, J. ZHANG, Q. ZHANG, AND Z. ZHANG, Fast evaluation of the Caputo fractional derivative and its applications to fractional diffusion equations, Comm. Comput. Phys., 21 (2017), pp. 650-678.

[16] B. Jin, R. LAZAROV, AND Z. ZHOU, Two fully discrete schemes for fractional diffusion and diffusion-wave equations with nonsmooth data, SIAM J. Sci. Comput., 38 (2016), pp. A146-A170.

[17] B. JIN, B. LI, AND Z. ZHOU, Numerical analysis of nonlinear subdiffusion equations, SIAM J. Numer. Anal., 56 (2018), pp. 1-23.

[18] A. A. Kilbas, H. M. SRivastava, and J. J. Trujillo, Theory and Applications of Fractional Differential Equations, vol. 204, Elsevier Science Limited, 2006.

[19] P. Kumar AND O. AgRAWAL, An approximate method for numerical solution of fractional differential equations, Signal Process., 86 (2006), pp. 2602-2610.

[20] C. LI, R. WU, AND H. DING, High-order approximation to Caputo derivatives and Caputotype advection-diffusion equations, Comm. Appl. Industrial Math., e-536 (2014), pp. 1-32.

[21] C. Li AND F. Zeng, Numerical Methods for Fractional Calculus, Chapman and Hall/CRC, 2015.

[22] D. LI, H.-L. LiaO, W. Sun, J. WANG, AND J. ZhANG, Analysis of L1-Galerkin FEMs for time-fractional nonlinear parabolic problems, Commun. Comput. Phys., 24 (2018), pp. 86103.

[23] H. LI, J. CAO, AND C. LI, High-order approximation to Caputo derivatives and Caputo-type advection-diffusion equations (iii), J. Comput. Appl. Math., 299 (2016), pp. 159-175.

[24] H.-L. LIAO, D. LI, AND J. ZHANG, Sharp error estimate of the nonuniform L1 formula for linear reaction-subdiffusion equations, SIAM J. Numer. Anal., 56 (2018), pp. 1112-1133.

[25] H.-L. LIAO, W. MCLEAN, AND J. ZHANG, A discrete gronwall inequality with applications to numerical schemes for subdiffusion problems, SIAM J. Numer. Anal., 57 (2019), pp. 218237.

[26] H.-L. LIAO, Y. YAN, AND J. ZHANG, Unconditional convergence of a fast two-level linearized algorithm for semilinear subdiffusion equations, J. Sci. Comput., 80 (2019), pp. 1-25.

[27] Y. LIN AND C. XU, Finite difference/spectral approximations for the time-fractional diffusion equation, J. Comput. Phys., 225 (2007), pp. 1533-1552.

[28] P. LinZ, Analytical and Numerical Methods for Volterra Equations, Society for Industrial Mathematics, Philadelphia, 1985.

[29] C. LuBICH, Discretized fractional calculus, SIAM J. Math. Anal., 17 (1986), pp. 704-719.

[30] Y. Luchko, F. Mainardi, AND Y. Povstenko, Propagation speed of the maximum of the fundamental solution to the fractional diffusion-wave equation, Comput. Math. with Appl., 66 (2013), pp. 774-784.

[31] W.-H. LuO, C. LI, T.-Z. Huang, X.-M. GU, AND G.-C. Wu, A high-order accurate numerical scheme for the Caputo derivative with applications to fractional diffusion problems, Numer. Func. Anal. Opt., 39 (2017), pp. 600-622.

[32] C. LV AND C. XU, Error analysis of a high order method for time-fractional diffusion equations, SIAM J. Sci. Comput., 38 (2016), pp. A2699-A2724.

[33] F. MAINARDI, Fractional Calculus and Waves in Linear Viscoelasticity: an Introduction to Mathematical Models, World Scientific, 2010.

[34] K. S. Miller AND B. Ross, An Introduction to the Fractional Calculus and Fractional Differential Equations, Wiley-Interscience, 1993.

[35] T. B. NGUYEN AND B. JANG, A high-order predictor-corrector method for solving nonlinear differential equations of fractional order, Frac. Cal. Appl. Anal., 20 (2017), pp. 447-476.

[36] M. STYNES, E. O'RioRDAN, AND J. L. GRACIA, Error analysis of a finite difference method 
on graded meshes for a time-fractional diffusion equation, SIAM J. Numer. Anal., 55 (2017), pp. 1057-1079.

[37] H. Sun, Z. SUn, AND R. Du, A linearized second-order difference scheme for the nonlinear time-fractional fourth-order reaction-diffusion equation, Numer. Math. Theor. Meth. Appl., 12 (2019), pp. 1168-1190.

[38] S. VONG, C. SHI, AND P. LYU, High-order compact schemes for fractional differential equations with mixed derivatives, Numer. Methods Partial Differential Equations, 33 (2017), pp. 2141-2158.

[39] Y. YAN, Z.-Z. SUN, AND J. ZHANG, Fast evaluation of the Caputo fractional derivative and its applications to fractional diffusion equations: A second-order scheme, Commun. Comput. Phys., 22 (2017), pp. 1028-1048.

[40] A. YounG, The application of approximate product-integration to the numerical solution of integral equations, Proc. Roy. Soc. London Ser. A, 224 (1954), pp. 561-573.

[41] F. ZENG, I. TURNER, AND K. BURRAGE, A stable fast time-stepping method for fractional integral and derivative operators, J. Sci. Comput., 77 (2018), pp. 283-307.

[42] F. ZENG, Z. ZHANG, AND G. E. KARNIADAKIS, Second-order numerical methods for multiterm fractional differential equations: smooth and non-smooth solutions, Comp. Methods Appl. Mech. Eng., 327 (2017), pp. 478-502. 